\title{
Impedance-based Gaussian Processes for Modeling Human Motor Behavior in Physical and Non-physical Interaction
}

\author{
José R. Medina, Hendrik Börner, Student Member, IEEE, \\ Satoshi Endo, and Sandra Hirche, Senior Member, IEEE
}

\begin{abstract}
Objective: Modeling of human motor intention plays an essential role in predictively controlling a robotic system in human-robot interaction tasks. In most machine learning techniques, human motor behavior is modeled as a generic stochastic process. However, the integration of a priori knowledge about underlying system structures can provide insights on otherwise unobservable intrinsic states that yield superior prediction performance and increased generalization capabilities. Methods: We present a novel method for modeling human motor behavior that explicitly includes a neuroscientifically supported model of human motor control, in which the dynamics of the human arm are modeled by a mechanical impedance that tracks a latent desired trajectory. We adopt a Bayesian setting by defining Gaussian process (GP) priors for the impedance elements and the latent desired trajectory. This enables exploitation of a priori human arm impedance knowledge for regression of interaction forces through inference of a latent desired human trajectory. Results: The method is validated using simulated data, with particular focus on effects of GP prior parameterization and intention estimation capabilities. Superior prediction performance is shown with respect to a naive GP prior. An experiment with human participants evaluates generalization capabilities and effects of training data sparsity. Conclusion: We derive the correlations of an impedance-based GP model of human motor behavior that exploits a priori knowledge. Significance: The model effectively predicts interaction forces by inferring a latent desired human trajectory in previously observed as well as unobserved regions of the input space.
\end{abstract}

Index Terms-Bayesian Modeling, Gaussian Processes, Human Motor Control, Human Motor Intention, Human Robot Interaction, Impedance, Kernel Structure

\section{INTRODUCTION}

C URRENT trends in robotics research are enabling direct robotic assistance through physical and non-physical human-robot interaction (HRI) in medical, industrial, and domestic domains. In HRI, knowledge of the human motor

Manuscript received August 13, 2018; revised October 25, 2018; accepted December 24, 2018. This research is partly supported by the ERC Starting Grant "Control based on Human Models (con-humo)" under grant agreement no. 337654. (Corresponding author: H. Börner.)

J. R. Medina and H. Börner contributed equally to this work.

J. R. Medina is with Franka Emika GmbH, 80797 Munich, Germany (e-mail: jose.medina@franka.de). H. Börner, S. Endo, and S. Hirche are with the Chair of Information-oriented Control, Department of Electrical and Computer Engineering, Technical University of Munich, 80333 Munich, Germany (e-mail: \{hendrik.boerner, s.endo, hirche\}@tum.de). intention is key to guarantee safety and comfort of the human. Moreover, it has significant impact on interaction performance. In the medical domain, the efficiency of physical assistance and rehabilitation greatly profits from a reliable prediction of the human motor intention [1]. In active exoskeleton systems, impedance controllers can exploit the additional information to provide anticipatory control signals [2]. In general HRI, imitation learning [3] and variable impedance control [4] are possible applications. However, due to the complexity of human motor behavior, i.a. caused by the redundancy of the musculoskeletal system [5] and neural noise [6], the estimation of human motor intention remains a significant challenge.

In human motor behavior modeling for HRI applications, sensory-motor control is generally treated as a "black box", for which inputs (e.g., task-related parameters and human body configurations) and outputs (e.g., interaction forces) are deterministically or stochastically mapped onto a model with no additional constraints (e.g., [7]). However, the integration of a priori knowledge about underlying behavioral structures can provide insights on otherwise unobservable intrinsic states, such as a goal point or a desired arm trajectory. Moreover, it can lead to superior prediction performance and generalization capabilities, as it guarantees that all correlations are inherently consistent with the structural constraints. Nonetheless, the exploitation of a priori knowledge in human motor behavior modeling remains an open issue with great potential.

In this article, we present a novel method for modeling human motor behavior in physical and non-physical HRI that explicitly incorporates a neuroscientifically supported model of human motor control. Without loss of generality, particular focus is placed on the derivation of a model for physical HRI, as it represents the more challenging problem. Motivated by relevance for envisaged applications in HRI, we consider a human arm motion of a complex trajectory and approximate the lower dimensional manifolds of human motor behavior by an impedance control scheme that counteracts deviations from a latent desired trajectory. We adopt a Bayesian setting by assuming Gaussian process (GP) priors for the impedance components and the latent desired trajectory. This enables the exploitation of a priori human arm impedance knowledge for the regression of human motor behavior. Moreover, it enables the estimation of human motor intention in the form of a desired trajectory with confidence levels. In order to facilitate 
proper parameterization of the GP priors, an overview of estimated human arm impedance characteristics is presented. The model is validated in simulation. The direct control over simulated intrinsic states enables both validation of intention estimation capabilities as well as assessment of influences of GP prior parameterization. Superior prediction performance is shown with respect to a naive GP prior. An experiment with human participants is conducted to analyze effects of training data sparsity and demonstrate generalization capabilities.

\section{A. Related work}

In current HRI research, human motor behavior is modeled in different ways. The authors of [8] propose a method for short-term motor behavior predictions based on observed forces and respective derivatives. Others transfer the minimum jerk principle [9] to HRI scenarios (e.g., [10] and [11]) for both short- and long-term predictions, depending on the type of the task. For tasks with increased complexity, the minimum jerk method is outperformed by a combination of judiciously defined polynomials that allow for more flexibility in the approximation of the dynamics [12]. In contrast to dynamics level methods, the programming by demonstration paradigm is applied for the derivation of long-term motor behavior predictions at task level. Demonstrations of task-specific HRI behavior are acquired through direct physical interaction [7] or teleoperation [13] and are encoded into a probabilistic model that captures the joint statistics of the observed trajectories and forces [14]. These models are naive in the sense that they do not include any a priori knowledge on the system. However, the inclusion of such knowledge can provide insights on unobservable intrinsic states and lead to superior prediction performance and generalization capabilities. GP-based models represent a powerful, non-parametric approach that allows for the integration of a priori knowledge and only requires the definition of second-order statistics between function values to efficiently approximate continuous functions from data [15]. They are well-suited for human motor behavior modeling, due to their desirable smoothness properties and their ability to model non-linear correlations. Thus, in this work, we include a model of human motor control, motivated by its relevance for envisaged applications in HRI, in a GP-based model.

The benefits of GPs for system identification are exploited for example in [16]-[21]. In [17], a GP is used to approximate a non-linear state vector field. In [18], an additional GP is used to model the non-linear mapping from the observation space to a latent state space. The resulting model is extended in [19], where human motion is modeled by explicitly incorporating an intention function into the latent state space transition function, which is modeled by a set of GPs. Second order interaction dynamics are explored in [20], where observed trajectories are considered to be driven by latent forces. In [21], a dynamic lower-dimensional representation of finger motion is obtained by decomposing variations in reach-and-grasp motions and modeling respective primitives with a multivariate GP model. GPs define input space correlations and derive predictions analytically by applying Bayes' rule, which yields confidence bounds of the results. These bounds are particularly interesting for control design, as they enable control adaptation according to the prediction confidence [22]. The performance of GPs depends on an appropriate kernel structure. If the structure of the correlation function is not defined in accordance with the real function, the convergence rate of the former towards the latter may increase exponentially. This leads to a decrease of the generalization capabilities in previously unobserved regions of the input space. The reliability and the validity of the prediction covariance as an indicator of the reliability decreases with increasing distance to the training data [23]. This dependency can be overcome by inclusion of a priori knowledge in the definition of the GP prior structure.

Human motor behavior is controlled by the central nervous system (CNS), which instantiates desired motor behavior through neural signals that regulate muscle activations at the limb joints. A theory of sensory-motor control proposes that these motor commands are appropriately scaled by an internal model [24], which is learned from experience and inverses the interaction dynamics. However, this feedforward component is subject to errors due to unexpected external forces [25], incomplete or incorrect internal models [26], and inherent neural noise [6]. A body of evidence suggests that, in order to counteract the resulting deviations, a combination of peripheral neuromuscular properties of the limb and motor commands from the CNS generate a feedback component that is directed towards the desired kinematic state and well-described by a mechanical impedance [27]. It consists of the impedance components inertia, damping, and stiffness and represents the effects of co-contraction of antagonistic muscles, involuntary reflexes, and intrinsic viscoelastic properties of the limb [28]. Thus, in this work, we assume GP priors for the impedance components and the desired trajectory. A preliminary work has been presented in [29]. In this article, we provide advanced theory by generalization to non-physical HRI. We present an overview of estimated human arm impedance characteristics and discuss implications for the GP prior parameterization. In order to consider the highly task-specific nature of human motor behavior in realistic HRI, we complement a validation of a time-dependent desired trajectory. An experiment with human participants is performed to assess the performance with real data. Additionally, the generalization capabilities of the model and the effects of training data sparsity are analyzed.

The remainder of this article is structured as follows: the considered problem of human motor behavior regression via human motor intention inference is derived in Section II. An introduction to GPs is presented in Section III and the GP-based model is defined in Section IV. An overview of estimated impedance characteristics and implications for GP prior parameterization are presented in Section V. The model is validated with simulated data in Section VI and evaluated with experimental data in Section VII. Section VIII contains discussions and conclusions are summarized in Section IX.

Notation: by convention, bold characters denote vectors and capital letters are used for matrices. The expression $\mathcal{N}(\boldsymbol{x} \mid \boldsymbol{\mu}, \Sigma)$ describes a Gaussian random variable defined over $\boldsymbol{x}$ with mean $\boldsymbol{\mu}$ and covariance $\Sigma$. E $[\boldsymbol{x}]$ and $\operatorname{Var}[\boldsymbol{x}]$ are expected value and variance of $\boldsymbol{x}$, respectively. The symbol $\mathbb{N}^{+}$denotes a natural positive integer. 


\section{Problem setting}

In this section, we derive a human motor behavior model for physical HRI. We assume the robot directly assists the human, as he/she tightly grasps the robot end-effector and executes a desired movement, e.g., for upper body rehabilitation [30]. In such a task, safe and efficient assistance requires reliable estimates of interaction forces and desired movement. Thus, we derive a model that provides these estimates, using only robot end-effector force/torque and position measurements. Additionally, we describe adjustments for non-physical HRI.

The rigid body dynamics of the human arm, when tightly grasping the robot end-effector, is given by

$$
\boldsymbol{\tau}_{\mathrm{hu}}+\boldsymbol{\tau}_{\mathrm{int}}=M_{q}(\boldsymbol{q}) \ddot{\boldsymbol{q}}+C(\boldsymbol{q}, \dot{\boldsymbol{q}}) \dot{\boldsymbol{q}}+\boldsymbol{g}(\boldsymbol{q}),
$$

where $\boldsymbol{q} \in \mathbb{R}^{7}$ is the 7 degree of freedom (DoF) configuration of the arm in joint space, $M_{q}(\boldsymbol{q}) \in \mathbb{R}^{7 \times 7}$ is the inertia matrix, $C(\boldsymbol{q}, \dot{\boldsymbol{q}}) \dot{\boldsymbol{q}} \in \mathbb{R}^{7}$ represents the Coriolis and centrifugal forces, $\boldsymbol{g}(\boldsymbol{q}) \in \mathbb{R}^{7}$ is the gravity vector, $\boldsymbol{\tau}_{\mathrm{hu}} \in \mathbb{R}^{7}$ are the human joint torques and $\tau_{\text {int }} \in \mathbb{R}^{7}$ are the interaction torques [25]. The interaction torques $\boldsymbol{\tau}_{\text {int }}=-J(\boldsymbol{q})^{\top} \boldsymbol{u}_{\text {int }}$, where $\boldsymbol{u}_{\text {int }} \in \mathbb{R}^{6}$ are the force/torque measurements and $J(\boldsymbol{q})$ is the Jacobian. The joint torques $\tau_{\text {hu }}$ consist of a feedforward term $\tau_{\mathrm{FF}}$, a feedback term $\tau_{\mathrm{FB}}$, and a neural noise term $\varepsilon_{\mathrm{N}, q}$, i.e.,

$$
\tau_{\mathrm{hu}}=\boldsymbol{\tau}_{\mathrm{FF}}+\boldsymbol{\tau}_{\mathrm{FB}}+\varepsilon_{\mathrm{N}, q},
$$

where $\varepsilon_{\mathrm{N}, q}$ represents the effects of joint torque noise, which depends on the muscle activation level [6], [31]. According to a theory of sensory-motor control, the feedforward term $\boldsymbol{\tau}_{\mathrm{FF}}$ contains inversed interaction dynamics of the musculoskeletal system, which are calculated with internal models previously learned by the CNS [24]. It specifies the joint torques that are necessary to attain the desired trajectory which we assume is a twice differentiable (with respect to time), well-defined function $\boldsymbol{q}_{d}(\boldsymbol{\theta}): \mathbb{R}^{n} \rightarrow \mathbb{R}^{7}$. As realistic human motor behavior in HRI is highly task-specific, the parameters $\boldsymbol{\theta} \in \mathbb{R}^{n}$ are given by suitable, task-specific input parameters such as the arm configuration $\boldsymbol{q}$, the angular velocities $\dot{\boldsymbol{q}}$, or the time $t$. In order to provide a comprehensive formulation, the model is first derived for a general desired trajectory $\boldsymbol{q}_{d}(\boldsymbol{\theta})$ and later analyzed for different parameterizations:

$$
\begin{aligned}
\boldsymbol{\tau}_{\mathrm{FF}}= & \hat{M}_{q}\left(\boldsymbol{q}_{d}(\boldsymbol{\theta})\right) \ddot{\boldsymbol{q}}_{d}(\boldsymbol{\theta})+\hat{C}\left(\boldsymbol{q}_{d}(\boldsymbol{\theta}), \dot{\boldsymbol{q}}_{d}(\boldsymbol{\theta})\right) \dot{\boldsymbol{q}}_{d}(\boldsymbol{\theta}) \\
& +\hat{\boldsymbol{g}}\left(\boldsymbol{q}_{d}(\boldsymbol{\theta})\right)-\hat{\boldsymbol{\tau}}_{\mathrm{int}}\left(\boldsymbol{q}_{d}(\boldsymbol{\theta}), \dot{\boldsymbol{q}}_{d}(\boldsymbol{\theta}), \ddot{\boldsymbol{q}}_{d}(\boldsymbol{\theta})\right)
\end{aligned}
$$

where the variables $\hat{\boldsymbol{\tau}}_{\text {int }}\left(\boldsymbol{q}_{d}(\boldsymbol{\theta}), \dot{\boldsymbol{q}}_{d}(\boldsymbol{\theta}), \ddot{\boldsymbol{q}}_{d}(\boldsymbol{\theta})\right), \hat{M}_{q}\left(\boldsymbol{q}_{d}(\boldsymbol{\theta})\right)$, $\hat{C}\left(\boldsymbol{q}_{d}(\boldsymbol{\theta}), \dot{\boldsymbol{q}}_{d}(\boldsymbol{\theta})\right)$, and $\hat{\boldsymbol{g}}\left(\boldsymbol{q}_{d}(\boldsymbol{\theta})\right)$ are the estimated counterparts of the respective variables in (1) [32]. According to a large body of evidence, the feedback term $\tau_{\mathrm{FB}}$ consists of restoring torques towards the desired trajectory $\boldsymbol{q}_{d}(\boldsymbol{\theta})$, which contain the effects of the impedance components: joint damping and joint stiffness. Although both components possess non-linear characteristics [5], the resulting feedback behavior can be described by a linear approximation [27]:

$$
\boldsymbol{\tau}_{\mathrm{FB}}=D_{q}(\boldsymbol{q}, \dot{\boldsymbol{q}}, \boldsymbol{\theta}) \dot{\boldsymbol{e}}_{q}(\boldsymbol{\theta})+K_{q}(\boldsymbol{q}, \dot{\boldsymbol{q}}, \boldsymbol{\theta}) \boldsymbol{e}_{q}(\boldsymbol{\theta}),
$$

where the joint error $\boldsymbol{e}_{q}(\boldsymbol{\theta})=\boldsymbol{q}_{d}(\boldsymbol{\theta})-\boldsymbol{q}$ and joint damping $D_{q}(\boldsymbol{q}, \dot{\boldsymbol{q}}, \boldsymbol{\theta}) \in \mathbb{R}^{7 \times 7}$ and joint stiffness $K_{q}(\boldsymbol{q}, \dot{\boldsymbol{q}}, \boldsymbol{\theta}) \in \mathbb{R}^{7 \times 7}$ depend on the arm configuration $\boldsymbol{q}$, the angular velocities $\dot{\boldsymbol{q}}$, and the task-specific input parameters $\boldsymbol{\theta}$. The former two dependencies are due to the length- and velocity-tension relationships of muscle fibers [25] and the latter dependency is a result of the influence of the muscle activation [32]. Substituting (2), (3), and (4) into (1) yields

$$
\begin{aligned}
-\boldsymbol{\tau}_{\mathrm{int}}= & M_{q}(\boldsymbol{q}) \ddot{\boldsymbol{e}}_{q}(\boldsymbol{\theta})+C(\boldsymbol{q}, \dot{\boldsymbol{q}}) \dot{\boldsymbol{e}}_{q}(\boldsymbol{\theta})+\boldsymbol{\varepsilon}_{\mathrm{dyn}, q}(\boldsymbol{q}, \dot{\boldsymbol{q}}, \ddot{\boldsymbol{q}}, \boldsymbol{\theta}) \\
& -\hat{\boldsymbol{\tau}}_{\mathrm{int}}\left(\boldsymbol{q}_{d}(\boldsymbol{\theta}), \dot{\boldsymbol{q}}_{d}(\boldsymbol{\theta}), \ddot{\boldsymbol{q}}_{d}(\boldsymbol{\theta})\right)+D_{q}(\boldsymbol{q}, \dot{\boldsymbol{q}}, \boldsymbol{\theta}) \dot{\boldsymbol{e}}_{q}(\boldsymbol{\theta}) \\
& +K_{q}(\boldsymbol{q}, \dot{\boldsymbol{q}}, \boldsymbol{\theta}) \boldsymbol{e}_{q}(\boldsymbol{\theta})+\boldsymbol{\varepsilon}_{\mathrm{N}, q},
\end{aligned}
$$

where $\varepsilon_{\mathrm{dyn}, q}(\boldsymbol{q}, \dot{\boldsymbol{q}}, \ddot{\boldsymbol{q}}, \boldsymbol{\theta})$ are the discrepancies of the inertia, Coriolis, centrifugal, and gravity terms between (1) and (3). The elements of $C(\boldsymbol{q}, \dot{\boldsymbol{q}}) \dot{\boldsymbol{q}}$ are $\mathcal{O}\left(\dot{q}^{2}\right)$. As its contributions are small compared to those of the other terms, we include it in $\varepsilon_{\mathrm{dyn}, q}(\boldsymbol{q}, \dot{\boldsymbol{q}}, \ddot{\boldsymbol{q}}, \boldsymbol{\theta})$. Furthermore, in order to consider adaptation of the estimated interaction torques $\hat{\boldsymbol{\tau}}_{\text {int }}\left(\boldsymbol{q}_{d}(\boldsymbol{\theta}), \dot{\boldsymbol{q}}_{d}(\boldsymbol{\theta}), \ddot{\boldsymbol{q}}_{d}(\boldsymbol{\theta})\right)$ in response to unpredictable environmental dynamics during task execution, we replace the purely feedforward term in (5) by $\hat{\boldsymbol{\tau}}_{\text {int }}(\boldsymbol{q}, \dot{\boldsymbol{q}}, \ddot{\boldsymbol{q}}, \boldsymbol{\theta})$, which depends on the kinematics $\boldsymbol{q}, \dot{\boldsymbol{q}}, \ddot{\boldsymbol{q}}$ and the task-specific input parameters $\theta$. This yields

$$
\begin{aligned}
-\boldsymbol{\tau}_{\mathrm{int}}= & M_{q}(\boldsymbol{q}) \ddot{\boldsymbol{e}}_{q}(\boldsymbol{\theta})-\hat{\boldsymbol{\tau}}_{\mathrm{int}}(\boldsymbol{q}, \dot{\boldsymbol{q}}, \ddot{\boldsymbol{q}}, \boldsymbol{\theta})+D_{q}(\boldsymbol{q}, \dot{\boldsymbol{q}}, \boldsymbol{\theta}) \dot{\boldsymbol{e}}_{q}(\boldsymbol{\theta}) \\
& +K_{q}(\boldsymbol{q}, \dot{\boldsymbol{q}}, \boldsymbol{\theta}) \boldsymbol{e}_{q}(\boldsymbol{\theta})+\boldsymbol{\varepsilon}_{\mathrm{dyn}, q}(\boldsymbol{q}, \dot{\boldsymbol{q}}, \ddot{\boldsymbol{q}}, \boldsymbol{\theta})+\boldsymbol{\varepsilon}_{\mathrm{N}, q} .(6)
\end{aligned}
$$

Motivated by transferability to envisaged applications in HRI, we express the dynamics of the hand in Cartesian space:

$$
\begin{aligned}
\boldsymbol{u}_{\text {int }}(\overline{\boldsymbol{\xi}}) & =\boldsymbol{u}_{\text {imp }}(\overline{\boldsymbol{\xi}})+\hat{\boldsymbol{u}}_{\mathrm{int}, \varepsilon}(\overline{\boldsymbol{\xi}})+\boldsymbol{\varepsilon}_{\mathrm{N}}, \\
\boldsymbol{u}_{\mathrm{imp}}(\overline{\boldsymbol{\xi}}) & =M(\boldsymbol{x}) \ddot{\boldsymbol{e}}(\boldsymbol{\theta})+D(\boldsymbol{\xi}) \dot{\boldsymbol{e}}(\boldsymbol{\theta})+K(\boldsymbol{\xi}) \boldsymbol{e}(\boldsymbol{\theta}),
\end{aligned}
$$

where $\boldsymbol{x} \in \mathbb{R}^{6}$ is the hand configuration in Cartesian space, defined by the forward kinematics mapping $L(\boldsymbol{q}): \mathbb{R}^{7} \rightarrow \mathbb{R}^{6}$. The error $\boldsymbol{e}(\boldsymbol{\theta})=\boldsymbol{x}_{d}(\boldsymbol{\theta})-\boldsymbol{x}$, in which $\boldsymbol{x}_{d}(\boldsymbol{\theta})$ is the desired trajectory, and $\hat{\boldsymbol{u}}_{\text {int }, \varepsilon}(\overline{\boldsymbol{\xi}})=\hat{\boldsymbol{u}}_{\text {int }}(\overline{\boldsymbol{\xi}})+\varepsilon_{\mathrm{dyn}}(\overline{\boldsymbol{\xi}})$. Additionally, $\boldsymbol{\xi}=\left[\boldsymbol{x}^{\top} \dot{\boldsymbol{x}}^{\top} \boldsymbol{\theta}^{\top}\right]^{\top}, \overline{\boldsymbol{\xi}}=\left[\boldsymbol{x}^{\top} \dot{\boldsymbol{x}}^{\top} \ddot{\boldsymbol{x}}^{\top} \boldsymbol{\theta}^{\top}\right]^{\top}, \boldsymbol{\varepsilon}_{\mathrm{N}}=J(\boldsymbol{q})^{-T} \varepsilon_{\mathrm{N}, q}$, and

$$
\begin{aligned}
M(\boldsymbol{x}) & =J(\boldsymbol{q})^{-T} M_{q}(\boldsymbol{q}) J(\boldsymbol{q})^{-1}, \\
D(\boldsymbol{\xi}) & =J(\boldsymbol{q})^{-T} D_{q}(\boldsymbol{q}, \dot{\boldsymbol{q}}, \boldsymbol{\theta}) J(\boldsymbol{q})^{-1}, \\
K(\boldsymbol{\xi}) & =J(\boldsymbol{q})^{-T} K_{q}(\boldsymbol{q}, \dot{\boldsymbol{q}}, \boldsymbol{\theta}) J(\boldsymbol{q})^{-1}, \\
\hat{\boldsymbol{u}}_{\mathrm{int}}(\overline{\boldsymbol{\xi}}) & =-J(\boldsymbol{q})^{-T} \hat{\boldsymbol{\tau}}_{\mathrm{int}}(\boldsymbol{q}, \dot{\boldsymbol{q}}, \ddot{\boldsymbol{q}}, \boldsymbol{\theta}), \\
\boldsymbol{\varepsilon}_{\mathrm{dyn}}(\overline{\boldsymbol{\xi}}) & =J(\boldsymbol{q})^{-T} \boldsymbol{\varepsilon}_{\mathrm{dyn}, q}(\boldsymbol{q}, \dot{\boldsymbol{q}}, \ddot{\boldsymbol{q}}, \boldsymbol{\theta}),
\end{aligned}
$$

In non-physical HRI, there are no interaction torques $\tau_{\text {int }}$. Thus, $\boldsymbol{\tau}_{\text {int }}=\mathbf{0}$ in (1), $\hat{\boldsymbol{\tau}}_{\text {int }}\left(\boldsymbol{q}_{d}(\boldsymbol{\theta}), \dot{\boldsymbol{q}}_{d}(\boldsymbol{\theta}), \ddot{\boldsymbol{q}}_{d}(\boldsymbol{\theta})\right)=\mathbf{0}$ in (3) and (5), and $\hat{\boldsymbol{\tau}}_{\text {int }}(\boldsymbol{q}, \dot{\boldsymbol{q}}, \ddot{\boldsymbol{q}}, \boldsymbol{\theta})=\mathbf{0}$ in (6). In Cartesian space, $\hat{\boldsymbol{u}}_{\text {int }}(\overline{\boldsymbol{\xi}})=\boldsymbol{u}_{\text {int }}(\overline{\boldsymbol{\xi}})=0$. Inserting these two correlations in (7) yields a variation of the model for non-physical HRI:

$$
\mathbf{0}=\boldsymbol{u}_{\mathrm{imp}}(\overline{\boldsymbol{\xi}})+\boldsymbol{\varepsilon}_{\mathrm{dyn}}(\overline{\boldsymbol{\xi}})+\boldsymbol{\varepsilon}_{\mathrm{N}} .
$$

As there are no interaction forces $\boldsymbol{u}_{\text {int }}(\overline{\boldsymbol{\xi}})$, this variation only requires position measurements. In this work, without loss of generality, particular focus is placed on the derivation of a human motor behavior model for physical HRI. As (9) is a simplification of (7), the model includes a simplified variation that applies to non-physical HRI. The problem considered in this work consists of the estimation of the interaction forces $\boldsymbol{u}_{\text {int }}(\overline{\boldsymbol{\xi}})$, based on the interaction dynamics in (7) and the inference of the human motor intention, represented by the desired trajectory $\boldsymbol{x}_{d}(\boldsymbol{\theta})$. This is to be achieved by means of regression analysis, given the input-output pairs $\left\{\overline{\boldsymbol{\xi}}, \boldsymbol{u}_{\text {int }}(\overline{\boldsymbol{\xi}})\right\}$. 


\section{GAUSSIAN PROCESS PRIORS}

A GP $f(\boldsymbol{z}): \mathbb{R}^{n} \rightarrow \mathbb{R}$ at input points $\boldsymbol{z} \in \mathbb{R}^{n}$ is a statistical distribution over function values, wherein any finite collection of samples $\left\{f\left(\boldsymbol{z}_{1}\right) \cdots f\left(\boldsymbol{z}_{h}\right)\right\}$ with number $h \in \mathbb{N}^{+}$forms a multivariate Gaussian random variable. A GP is compactly formulated as $f(\boldsymbol{z}) \sim \mathcal{G P}\left(m(\boldsymbol{z}), k\left(\boldsymbol{z}, \boldsymbol{z}^{\prime}\right)\right)$ and fully defined by its mean $m(\boldsymbol{z})=\mathrm{E}[f(\boldsymbol{z})]$ and covariance function

$$
\begin{aligned}
k\left(\boldsymbol{z}, \boldsymbol{z}^{\prime}\right) & =\operatorname{Cov}\left[\Delta f(\boldsymbol{z}), \Delta f\left(\boldsymbol{z}^{\prime}\right)\right] \\
& \left.=\mathrm{E}\left[(f(\boldsymbol{z})-m(\boldsymbol{z}))\left(f\left(\boldsymbol{z}^{\prime}\right)-m\left(\boldsymbol{z}^{\prime}\right)\right)^{\top}\right)\right],
\end{aligned}
$$

which represents the correlation of two input points $\left(\boldsymbol{z}, \boldsymbol{z}^{\prime}\right)$.

GPs possess the desirable properties of multivariate normal distributions, which enable the computation of conditional distributions. The joint prior distribution $\mathrm{P}\left(\boldsymbol{y}, y_{*} \mid Z, \boldsymbol{z}_{*}\right)$ of a given training set of noisy observations $\boldsymbol{y}=\left\{y_{j}\right\}_{j=1}^{h}$ at inputs $Z=\left\{\boldsymbol{z}_{j}\right\}_{j=1}^{h}$ and predictive output $y_{*}$ at test input $\boldsymbol{z}_{*}$ is

$$
\begin{aligned}
& \mathrm{P}\left(\boldsymbol{y}, y_{*} \mid Z, \boldsymbol{z}_{*}\right)= \\
& \mathcal{N}\left(\left[\begin{array}{c}
\boldsymbol{y} \\
y_{*}
\end{array}\right] \mid\left[\begin{array}{c}
m(Z) \\
m\left(\boldsymbol{z}_{*}\right)
\end{array}\right],\left[\begin{array}{cc}
K+\sigma_{n}^{2} I & \boldsymbol{k}_{*} \\
\boldsymbol{k}_{*}^{\top} & k_{* *}+\sigma_{n}^{2}
\end{array}\right]\right),
\end{aligned}
$$

where $K=k(Z, Z), \quad \boldsymbol{k}_{*}=k\left(\boldsymbol{z}_{*}, Z\right)$, and $k_{* *}=k\left(\boldsymbol{z}_{*}, \boldsymbol{z}_{*}\right)$ represent the covariances of the training inputs $Z$ and the test input $\boldsymbol{z}_{*}$. The matrix $I$ is the identity matrix and $\sigma_{n}^{2}$ is the observation noise variance. By means of multivariate Gaussian conditioning, i.e., application of Bayes' rule, the conditional (predictive) posterior $\mathrm{P}\left(y_{*} \mid \boldsymbol{y}, Z, \boldsymbol{z}_{*}\right)$ is

$$
\begin{gathered}
\mathrm{P}\left(y_{*} \mid \boldsymbol{y}, Z, \boldsymbol{z}_{*}\right)=\mathcal{N}\left(y_{*} \mid m\left(\boldsymbol{z}_{*}\right)+\boldsymbol{k}_{*}^{\top} K_{\sigma_{n}}^{-1}(\boldsymbol{y}-m(Z)),\right. \\
\left.k_{* *}+\sigma_{n}^{2}-\boldsymbol{k}_{*}^{\top} K_{\sigma_{n}}^{-1} \boldsymbol{k}_{*}\right),
\end{gathered}
$$

where $K_{\sigma_{n}}=K+\sigma_{n}^{2} I$. The computational load is governed by matrix inversion $\left(K+\sigma_{n}^{2} I\right)^{-1}$ with complexity $\mathcal{O}\left(h^{3}\right)$.

\section{BAYESIAN IMPEDANCE MODEL}

In this section, we derive a multi-layered Bayesian model, which is presented schematically in Fig. 1. The lowest layer contains the latent variables $M(\boldsymbol{x}), D(\boldsymbol{\xi}), K(\boldsymbol{\xi})$, and $\boldsymbol{x}_{d}(\boldsymbol{\theta})$. By assuming prior distributions on these latent variables, we construct a compound kernel for the impedance force $\boldsymbol{u}_{\mathrm{imp}}(\overline{\boldsymbol{\xi}})$. It forms a part of the middle layer, which additionally contains the neural noise variable $\varepsilon_{\mathrm{N}}$ and the latent variable $\hat{\boldsymbol{u}}_{\mathrm{int}, \varepsilon}(\overline{\boldsymbol{\xi}})$. By again assuming prior distributions, we combine all of the middle layer components to construct a compound kernel for the interaction force $\boldsymbol{u}_{\text {int }}(\overline{\boldsymbol{\xi}})$. It constitutes the top layer of our model and contains components that account for predictable $\left(\hat{\boldsymbol{u}}_{\mathrm{int}, \varepsilon}(\overline{\boldsymbol{\xi}})\right)$ and unpredictable $\left(\boldsymbol{u}_{\mathrm{imp}}(\overline{\boldsymbol{\xi}})\right)$ interaction dynamics. Due to dependency on $\bar{\xi}$, both components can be adapted during task execution. Given a training set $\left\{\boldsymbol{u}_{\text {int }, j}(\overline{\boldsymbol{\xi}}), \overline{\boldsymbol{\xi}}_{j}\right\}_{j=1}^{h}$, the model provides predictions $\boldsymbol{u}_{\text {int }}\left(\overline{\boldsymbol{\xi}}_{*}\right)$ at test input $\overline{\boldsymbol{\xi}}_{*}$ and infers the desired trajectory $\boldsymbol{x}_{d}\left(\boldsymbol{\theta}_{*}\right)$. The incorporation of the latter requires that the desired behavior can be described by a twice differentiable, well-defined function $\boldsymbol{q}_{d}(\boldsymbol{\theta})$, which is defined by suitable, task-specific input parameters $\theta$.

In this work, we approximate the variables $M(\boldsymbol{x}), D(\boldsymbol{\xi})$, and $K(\boldsymbol{\xi})$ in (8) by diagonal matrices. Consequently,

$$
\begin{aligned}
u_{\mathrm{int}, i}(\overline{\boldsymbol{\xi}}) & =u_{\mathrm{imp}, i}(\overline{\boldsymbol{\xi}})+\hat{u}_{\mathrm{int}, \varepsilon, i}(\overline{\boldsymbol{\xi}})+\varepsilon_{\mathrm{N}}, \\
u_{\mathrm{imp}, i}(\overline{\boldsymbol{\xi}}) & =m_{i} \ddot{e}_{i}(\boldsymbol{\theta})+d_{i}(\boldsymbol{\xi}) \dot{e}_{i}(\boldsymbol{\theta})+k_{i}(\boldsymbol{\xi}) e_{i}(\boldsymbol{\theta}),
\end{aligned}
$$

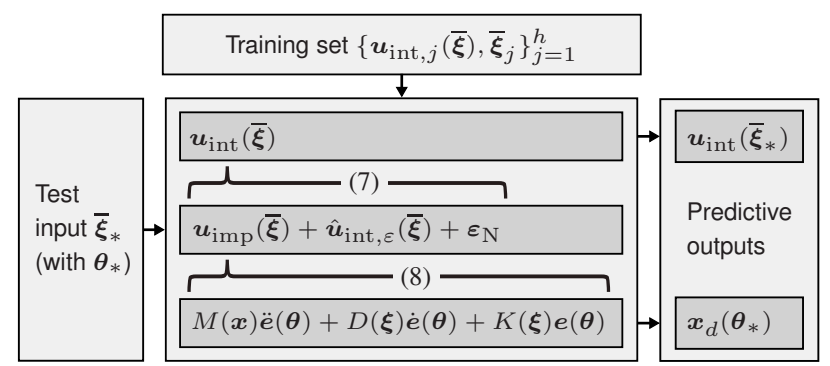

Fig. 1. Schematic block diagram of the Bayesian model. The transitions between the top, middle, and bottom layers correspond to (7) and (8).

where $e_{i}(\boldsymbol{\theta})=x_{d, i}(\boldsymbol{\theta})-x_{i}, u_{\mathrm{int}, i}(\overline{\boldsymbol{\xi}}), u_{\mathrm{imp}, i}(\overline{\boldsymbol{\xi}}), \hat{u}_{\mathrm{int}, \varepsilon, i}(\overline{\boldsymbol{\xi}})$, $d_{i}(\boldsymbol{\xi}), k_{i}(\boldsymbol{\xi}), x_{d, i}(\boldsymbol{\theta})$, and $x_{i}$ are one-dimensional functions, $m_{i}$ is a one-dimensional variable, and $\varepsilon_{\mathrm{N}} \sim \mathcal{N}\left(\varepsilon_{\mathrm{N}} \mid 0, \sigma_{\mathrm{N}}^{2}\right)$. Veridical biomechanical modeling requires inclusion of the non-diagonal elements of variables $M(\boldsymbol{x}), D(\boldsymbol{\xi})$, and $K(\boldsymbol{\xi})$. Although this is in principle possible in the context of the Bayesian impedance model, we approximate these variables by diagonal matrices. This approximation is motivated by multiple reasons: 1) Of the few quantitative Cartesian space results of human arm impedance estimation during movement, most are one-dimensional [33], [34] or assume decoupled arm dynamics [35]-[37]. 2) Non-diagonal elements would require convolved multi-output GPs with output correlations, which possess significantly higher computational complexity [38]. 3) These output correlations would significantly decrease the comprehensibility of the derivations and the analyses of effects of GP prior parameterization. For completeness, we discuss adjustments for multi-dimensional treatment in Section VIII.

\section{A. Impedance model priors}

For statistical analysis of (10), we assume that variable $m_{i}$ and functionals $d_{i}(\boldsymbol{\xi}), k_{i}(\boldsymbol{\xi}), x_{d, i}(\boldsymbol{\theta})$, and $\hat{u}_{\text {int }, \varepsilon, i}(\overline{\boldsymbol{\xi}})$ are all statistically independent and possess prior distributions

$$
\begin{aligned}
m_{i} & \sim \mathcal{N}\left(m_{i} \mid \mu_{m, i}, \sigma_{m, i}^{2}\right), \\
d_{i}(\boldsymbol{\xi}) & \sim \mathcal{G P}\left(\mu_{d, i}, k_{d}\left(\boldsymbol{\xi}, \boldsymbol{\xi}^{\prime}\right)\right), \\
k_{i}(\boldsymbol{\xi}) & \sim \mathcal{G P}\left(\mu_{k, i}, k_{k}\left(\boldsymbol{\xi}, \boldsymbol{\xi}^{\prime}\right)\right), \\
x_{d, i}(\boldsymbol{\theta}) & \sim \mathcal{G P}\left(\mu_{x_{d}, i}, k_{x_{d}}\left(\boldsymbol{\theta}, \boldsymbol{\theta}^{\prime}\right)\right), \\
\hat{u}_{\mathrm{int}, \varepsilon, i}(\overline{\boldsymbol{\xi}}) & \sim \mathcal{G} \mathcal{P}\left(0, k_{\widehat{\mathrm{int}}}\left(\overline{\boldsymbol{\xi}}, \overline{\boldsymbol{\xi}}^{\prime}\right)\right),
\end{aligned}
$$

where $\mu_{m, i}, \mu_{d, i}, \mu_{k, i}$, and $\mu_{x_{d}, i}$ are the respective expected mean values (EMVs) and $\sigma_{m, i}^{2}$ is the inertia signal variance. The priors are defined by the covariance functions $k_{d}\left(\boldsymbol{\xi}, \boldsymbol{\xi}^{\prime}\right)$, $k_{k}\left(\boldsymbol{\xi}, \boldsymbol{\xi}^{\prime}\right), k_{x_{d}}\left(\boldsymbol{\theta}, \boldsymbol{\theta}^{\prime}\right)$, and $k_{\widehat{\mathrm{int}}}\left(\overline{\boldsymbol{\xi}}, \overline{\boldsymbol{\xi}}^{\prime}\right)$, of which $k_{x_{d}}\left(\boldsymbol{\theta}, \boldsymbol{\theta}^{\prime}\right)$ is twice differentiable with respect to the task-specific input parameters $\boldsymbol{\theta}$. Due to the statistical independence, positive correlation of damping $d_{i}(\boldsymbol{\xi})$ and stiffness $k_{i}(\boldsymbol{\xi})$ [32] is not directly included in the priors. However, it can be partially incorporated by suitable definition of the EMVs $\mu_{d, i}$ and $\mu_{k, i}$. Positivity of damping $d_{i}(\boldsymbol{\xi})$ and stiffness $k_{i}(\boldsymbol{\xi})$ can be strictly ensured by means of warped GP priors [39], in which the priors are transformed to $\log \left(d_{i}(\boldsymbol{\xi})\right) \sim \mathcal{G} \mathcal{P}\left(\log \mu_{d, i}, k_{d}\left(\boldsymbol{\xi}, \boldsymbol{\xi}^{\prime}\right)\right)$ and $\log \left(k_{i}(\boldsymbol{\xi})\right) \sim \mathcal{G P}\left(\log \mu_{k, i}, k_{k}\left(\boldsymbol{\xi}, \boldsymbol{\xi}^{\prime}\right)\right)$. Application of these warped GP priors would require all kernel derivations to consider the $\log$ formulations and the respective derivatives. 
For better comprehensibility of the derivations, we instead assume that the respective signal variances only assign high probabilities to regions in the positive domain.

\section{B. Impedance, $P D$, and interaction force kernels}

Given (12), the terms $d_{i}(\boldsymbol{\xi}) \dot{e}_{i}(\boldsymbol{\theta})$ and $k_{i}(\boldsymbol{\xi}) e_{i}(\boldsymbol{\theta})$ in (11) are products of statistically independent Gaussian distributions. Consequently, the impedance force $u_{\mathrm{imp}, i}(\overline{\boldsymbol{\xi}})$ is a combination of both Gaussian and chi-squared distributions and possesses non-central chi-squared terms. Nonetheless, the computation of its expected value and covariance function enables its approximation as a GP. The corresponding loss of accuracy increases with the variances of the priors. Thus, by considering low variances due to proper parameterization, we are able to approximate the impedance force $u_{\mathrm{imp}, i}(\overline{\boldsymbol{\xi}})$ as a GP:

$$
u_{\mathrm{imp}, i}(\overline{\boldsymbol{\xi}}) \sim \mathcal{G} \mathcal{P}\left(\mathrm{E}\left[u_{\mathrm{imp}, i}(\overline{\boldsymbol{\xi}})\right], k_{\mathrm{imp}}\left(\overline{\boldsymbol{\xi}}, \overline{\boldsymbol{\xi}}^{\prime}\right)\right) .
$$

From (11) and (12), the expected value is

$$
\begin{aligned}
\mathrm{E}\left[u_{\mathrm{imp}, i}(\overline{\boldsymbol{\xi}})\right]= & \mu_{m, i}\left(\ddot{\mu}_{x_{d}, i}-\ddot{x}_{i}\right)+\mu_{d, i}\left(\dot{\mu}_{x_{d}, i}-\dot{x}_{i}\right) \\
& +\mu_{k, i}\left(\mu_{x_{d}, i}-x_{i}\right) .
\end{aligned}
$$

The expression for the impedance kernel

$$
\begin{aligned}
k_{\mathrm{imp}}\left(\overline{\boldsymbol{\xi}}, \overline{\boldsymbol{\xi}}^{\prime}\right)= & \operatorname{Cov}\left[m_{i} \ddot{e}_{i}(\boldsymbol{\theta})+d_{i}(\boldsymbol{\xi}) \dot{e}_{i}(\boldsymbol{\theta})+k_{i}(\boldsymbol{\xi}) e_{i}(\boldsymbol{\theta}),\right. \\
& \left.m_{i} \ddot{e}_{i}\left(\boldsymbol{\theta}^{\prime}\right)+d_{i}\left(\boldsymbol{\xi}^{\prime}\right) \dot{e}_{i}\left(\boldsymbol{\theta}^{\prime}\right)+k_{i}\left(\boldsymbol{\xi}^{\prime}\right) e_{i}\left(\boldsymbol{\theta}^{\prime}\right)\right]
\end{aligned}
$$

is more involved due to the correlations with the desired trajectory $x_{d, i}(\boldsymbol{\theta})$. As differentiation is a linear operator, the derivatives $\dot{x}_{d, i}(\boldsymbol{\theta})$ and $\ddot{x}_{d, i}(\boldsymbol{\theta})$ are also GPs that possess time derivative covariance functions [17]. Based on the covariances of sums and products [40], the impedance kernel $k_{\mathrm{imp}}\left(\overline{\boldsymbol{\xi}}, \overline{\boldsymbol{\xi}}^{\prime}\right)$ is the sum of the covariances of all product term combinations. For simplicity, we first consider only the terms that include damping $d_{i}(\boldsymbol{\xi})$ and stiffness $k_{i}(\boldsymbol{\xi})$ in (18), which corresponds to proportional-derivative (PD) control. The PD kernel

$$
\begin{gathered}
k_{\mathrm{PD}}\left(\boldsymbol{\xi}, \boldsymbol{\xi}^{\prime}\right)=\operatorname{Cov}\left[k_{e, i}(\boldsymbol{\xi}), k_{e, i}\left(\boldsymbol{\xi}^{\prime}\right)\right]+\operatorname{Cov}\left[k_{e, i}(\boldsymbol{\xi}), d_{\dot{e}, i}\left(\boldsymbol{\xi}^{\prime}\right)\right] \\
+\operatorname{Cov}\left[d_{\dot{e}, i}(\boldsymbol{\xi}), k_{e, i}\left(\boldsymbol{\xi}^{\prime}\right)\right]+\operatorname{Cov}\left[d_{\dot{e}, i}(\boldsymbol{\xi}), d_{\dot{e}, i}\left(\boldsymbol{\xi}^{\prime}\right)\right],
\end{gathered}
$$

wherein $k_{e, i}(\boldsymbol{\xi})=k_{i}(\boldsymbol{\xi}) e_{i}(\boldsymbol{\theta})$ and $d_{\dot{e}, i}(\boldsymbol{\xi})=d_{i}(\boldsymbol{\xi}) \dot{e}_{i}(\boldsymbol{\theta})$. For brevity, notations $k_{x_{d}}\left(\boldsymbol{\theta}, \boldsymbol{\theta}^{\prime}\right), k_{k}\left(\boldsymbol{\xi}, \boldsymbol{\xi}^{\prime}\right)$, and $k_{d}\left(\boldsymbol{\xi}, \boldsymbol{\xi}^{\prime}\right)$ are abbreviated by $k_{x_{d}}, k_{k}$, and $k_{d}$ in the remainder of the kernel derivation process. Inclusion of priors (12) in (16) yields

$$
\begin{aligned}
& k_{\mathrm{PD}}\left(\boldsymbol{\xi}, \boldsymbol{\xi}^{\prime}\right)=\mu_{k, i} \mu_{d, i}\left(\frac{\partial k_{x_{d}}}{\partial t^{\prime}}+\frac{\partial k_{x_{d}}}{\partial t}\right)+\left(\mu_{d, i}^{2}+k_{d}\right) \frac{\partial^{2} k_{x_{d}}}{\partial t \partial t^{\prime}} \\
& \quad+\left(\mu_{k, i}^{2}+k_{k}\right) k_{x_{d}}+\left(\dot{\mu}_{x_{d}, i}-\dot{x}_{i}\right)\left(\dot{\mu}_{x_{d}, i}-\dot{x}_{i}^{\prime}\right) k_{d} \\
& \quad+\left(\mu_{x_{d}, i}-x_{i}\right)\left(\mu_{x_{d}, i}-x_{i}^{\prime}\right) k_{k}
\end{aligned}
$$

where $t$ and $t^{\prime}$ belong to observations $\boldsymbol{\theta}$ and $\boldsymbol{\theta}^{\prime}$, respectively. Considering the inertia $m_{i}$ yields the impedance kernel

$$
\begin{aligned}
& k_{\mathrm{imp}}\left(\overline{\boldsymbol{\xi}}, \overline{\boldsymbol{\xi}}^{\prime}\right)=k_{\mathrm{PD}}\left(\boldsymbol{\xi}, \boldsymbol{\xi}^{\prime}\right)+\left(\ddot{\mu}_{x_{d}, i}-\ddot{x}_{i}\right)\left(\ddot{\mu}_{x_{d}, i}-\ddot{x}_{i}^{\prime}\right) \sigma_{m, i}^{2} \\
& +\left(\mu_{m, i}^{2}+\sigma_{m, i}^{2}\right) \frac{\partial^{4} k_{x_{d}}}{\partial t^{2} \partial t^{\prime 2}}+\mu_{d, i} \mu_{m, i}\left(\frac{\partial^{3} k_{x_{d}}}{\partial t^{\prime 2} \partial t}+\frac{\partial^{3} k_{x_{d}}}{\partial t^{2} \partial t^{\prime}}\right) \\
& +\mu_{k, i} \mu_{m, i}\left(\frac{\partial^{2} k_{x_{d}}}{\partial t^{\prime 2}}+\frac{\partial^{2} k_{x_{d}}}{\partial t^{2}}\right) .
\end{aligned}
$$

If $\boldsymbol{\theta}=t$, calculation of the time derivatives is straightforward. For alternative parameterizations, the time derivatives are

$$
\begin{aligned}
\frac{\partial k_{x_{d}}}{\partial t^{\prime}} & =\left(\frac{\partial k_{x_{d}}}{\partial \boldsymbol{\theta}^{\prime}}\right)^{\top} \frac{\partial \boldsymbol{\theta}^{\prime}}{\partial t}, \\
\frac{\partial^{2} k_{x_{d}}}{\partial t^{\prime 2}} & =\left(\frac{\partial \boldsymbol{\theta}^{\prime}}{\partial t}\right)^{\top} \frac{\partial^{2} k_{x_{d}}}{\partial \boldsymbol{\theta}^{\prime 2}} \frac{\partial \boldsymbol{\theta}^{\prime}}{\partial t}+\left(\frac{\partial k_{x_{d}}}{\partial \boldsymbol{\theta}^{\prime}}\right)^{\top} \frac{\partial^{2} \boldsymbol{\theta}^{\prime}}{\partial t^{2}} .
\end{aligned}
$$

Proposition 1: Assume the kernels $k_{d}\left(\boldsymbol{\xi}, \boldsymbol{\xi}^{\prime}\right), k_{k}\left(\boldsymbol{\xi}, \boldsymbol{\xi}^{\prime}\right)$, and $k_{x_{d}}\left(\boldsymbol{\theta}, \boldsymbol{\theta}^{\prime}\right)$ in (12) are symmetric and positive semi-definite and the desired trajectory kernel $k_{x_{d}}\left(\boldsymbol{\theta}, \boldsymbol{\theta}^{\prime}\right)$ is a twice differentiable function (with respect to the task-specific input parameters $\boldsymbol{\theta}$ ). Then, the PD kernel $k_{\mathrm{PD}}\left(\boldsymbol{\xi}, \boldsymbol{\xi}^{\prime}\right)$ in (17) and the impedance kernel $k_{\text {imp }}\left(\overline{\boldsymbol{\xi}}, \overline{\boldsymbol{\xi}}^{\prime}\right)$ in (18) are valid kernels.

Proof: A symmetric, positive semi-definite function $k(x, y)$ is a valid kernel function [41]. Thus, given the assumptions in the proposition, the kernels $k_{d}\left(\boldsymbol{\xi}, \boldsymbol{\xi}^{\prime}\right), k_{k}\left(\boldsymbol{\xi}, \boldsymbol{\xi}^{\prime}\right)$, and $k_{x_{d}}\left(\boldsymbol{\theta}, \boldsymbol{\theta}^{\prime}\right)$ are valid kernels. Given the desired trajectory kernel $k_{x_{d}}\left(\boldsymbol{\theta}, \boldsymbol{\theta}^{\prime}\right)$ is a twice differentiable function, the PD kernel $k_{\mathrm{PD}}\left(\boldsymbol{\xi}, \boldsymbol{\xi}^{\prime}\right)$ and the impedance kernel $k_{\text {imp }}\left(\bar{\xi}, \overline{\boldsymbol{\xi}}^{\prime}\right)$ are defined by the covariance functions (17) and (18), respectively. If a compound kernel is defined by affine transformations of valid kernels, it is also a valid kernel [42]. As the covariance functions (17) and (18) are only defined by affine transformations of the kernels $k_{d}\left(\boldsymbol{\xi}, \boldsymbol{\xi}^{\prime}\right)$, $k_{k}\left(\boldsymbol{\xi}, \boldsymbol{\xi}^{\prime}\right)$, and $k_{x_{d}}\left(\boldsymbol{\theta}, \boldsymbol{\theta}^{\prime}\right)$, the PD kernel $k_{\mathrm{PD}}\left(\boldsymbol{\xi}, \boldsymbol{\xi}^{\prime}\right)$ and the impedance kernel $k_{\mathrm{imp}}\left(\overline{\boldsymbol{\xi}}, \overline{\boldsymbol{\xi}}^{\prime}\right)$ are valid kernels.

With (10) and (12), the a priori statistical characterization of the interaction force $u_{\mathrm{int}, i}(\overline{\boldsymbol{\xi}})$ is given by

$$
u_{\mathrm{int}, i}(\overline{\boldsymbol{\xi}}) \sim \mathcal{G P}\left(\mathrm{E}\left[u_{\mathrm{imp}, i}(\overline{\boldsymbol{\xi}})\right], k_{\mathrm{int}}\left(\overline{\boldsymbol{\xi}}, \overline{\boldsymbol{\xi}}^{\prime}\right)\right),
$$

where $\mathrm{E}\left[u_{\mathrm{int}, i}(\overline{\boldsymbol{\xi}})\right]=\mathrm{E}\left[u_{\mathrm{imp}, i}(\overline{\boldsymbol{\xi}})\right]$, because the EMV of prior $\hat{u}_{\text {int }, \varepsilon, i}(\overline{\boldsymbol{\xi}})$ in (12) is zero, and the interaction force kernel

$$
k_{\text {int }}\left(\overline{\boldsymbol{\xi}}, \overline{\boldsymbol{\xi}}^{\prime}\right)=k_{\text {imp }}\left(\overline{\boldsymbol{\xi}}, \overline{\boldsymbol{\xi}}^{\prime}\right)+k_{\widehat{\text { int }}}\left(\overline{\boldsymbol{\xi}}, \overline{\boldsymbol{\xi}}^{\prime}\right)+\sigma_{\mathrm{N}}^{2} .
$$

Proper parameterization of prior $x_{d, i}(\boldsymbol{\theta})$ is key, as it effects all impedance terms. Given a priori task-specific information, its EMV $\mu_{x_{d}, i}$ may be defined as a function of time or other task-specific input parameters. As deviations from the desired trajectory $x_{d, i}(\boldsymbol{\theta})$ are compensated through impedance control, the overall mean of the current state $x_{i}$ approximates the desired trajectory state $x_{d, i}(\boldsymbol{\theta})$, i.e., the steady state, in which the human is at "equilibrium". In HRI scenarios, where a priori task-specific information is not available, this relationship can be incorporated by defining the desired trajectory EMV $\mu_{x_{d}, i}$ by the current state $x_{i}$. With this definition, the prior

$$
x_{d, i}(\boldsymbol{\theta}) \sim \mathcal{G P}\left(x_{i}, k_{x_{d}}\left(\boldsymbol{\theta}, \boldsymbol{\theta}^{\prime}\right)\right) .
$$

Inserting (21) in (14) results in $\mathrm{E}\left[u_{\mathrm{imp}, i}(\overline{\boldsymbol{\xi}})\right]=0$, which, when inserted in (19), results in $\mathrm{E}\left[u_{\mathrm{int}, i}(\overline{\boldsymbol{\xi}})\right]=0$.

The derived model includes a non-physical HRI variation that corresponds to (9). For this variation, the priors in (12) do not include the estimated interaction force $\hat{u}_{\text {int }, \varepsilon, i}(\overline{\boldsymbol{\xi}})$ and the dynamic discrepancy $\varepsilon_{\text {dyn }} \sim \mathcal{N}\left(\varepsilon_{\text {dyn }} \mid 0, \sigma_{\text {dyn }}^{2}\right)$. Additionally, the interaction force kernel $k_{\text {int }}\left(\overline{\boldsymbol{\xi}}, \overline{\boldsymbol{\xi}}^{\prime}\right)$ in (19) is replaced by

$$
\bar{k}_{\text {int }}\left(\overline{\boldsymbol{\xi}}, \overline{\boldsymbol{\xi}}^{\prime}\right)=k_{\mathrm{imp}}\left(\overline{\boldsymbol{\xi}}, \overline{\boldsymbol{\xi}}^{\prime}\right)+\sigma_{\mathrm{dyn}}^{2}+\sigma_{\mathrm{N}}^{2} .
$$

Apart from these differences, all derivations in this section, including (17) and (18), are identical and equally valid. 

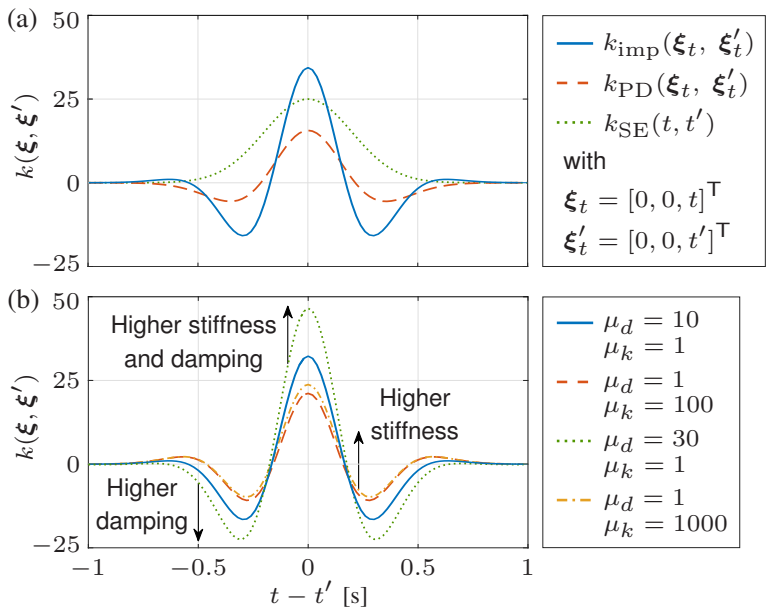

Fig. 2. Covariance functions with $\boldsymbol{\theta}=\boldsymbol{t}$. (a) Impedance, PD, and SE kernel with hyperparameters $\{0.1,(0.2)\}_{x_{d}},\{0.1,(0.10 .1)\}_{d}$ $\{0.1,(0.10 .1)\}_{k}$, and $\sigma_{m}^{2}=0.1$. The EMVs are $\mu_{m}=1 \mathrm{~kg}$ $\mu_{d}=10 \mathrm{Ns} / \mathrm{m}, \mu_{k}=100 \mathrm{Nm}$, and $\mu_{x_{d}}=0 \mathrm{~m}$. (b) Impedance kernel with identical parameters, apart from different EMVs $\mu_{d}$ and $\mu_{k}$.

For illustrative purposes, from here on, let all kernels in (12) be defined as Squared Exponential (SE) kernels

$$
k_{\mathrm{SE}}\left(\boldsymbol{z}, \boldsymbol{z}^{\prime}\right)=\sigma_{f, i}^{2} \exp \left\{-\left(\boldsymbol{z}-\boldsymbol{z}^{\prime}\right)^{\top} \Lambda_{i}^{-1}\left(\boldsymbol{z}-\boldsymbol{z}^{\prime}\right)\right\}
$$

with hyperparameters $\left\{\sigma_{f, i}^{2}, \Lambda_{i}\right\}_{\mathrm{SE}}$, where $\Lambda_{i}$ contains the length scales of the input dimensions $l_{1, i} \cdots l_{n, i}$ and $\sigma_{f, i}^{2}$ is the signal variance. The SE kernel is the most common kernel due to its desirable smoothness and convergence properties. As it is both infinitely differentiable and a valid kernel [23], it satisfies all of the prerequisites listed in Proposition 1.

In order to illustrate the effects of the terms comprising time derivatives of the desired trajectory, Fig. 2 shows covariance functions of a SE, a PD, and an impedance kernel for a time-dependent desired trajectory, i.e., $\boldsymbol{\theta}=t$. The PD kernel considers the derivative of the SE kernel as an additive term that governs its profile. Similarly, the profile of the impedance kernel is governed by the inertia term. The effects of the stiffness, damping, and inertia terms on the profiles of the respective kernels are significantly influenced by their EMVs. This dependency is illustrated in Fig. 2b, which shows several impedance covariance functions defined by different EMVs. As the stiffness term is governed by a SE kernel, it solely adds positive values with unnormalized Gaussian shape. Therefore, the higher the stiffness EMV, the higher the overall correlation. In contrast, increasing damping or inertia EMVs emphasizes the profiles of the first or second derivative, respectively.

In order to illustrate these effects for a model with a desired trajectory that is not defined by time, Fig. 3 shows covariance functions of a PD kernel for a configuration-dependent desired trajectory, i.e., $\boldsymbol{\theta}=x$. When the input point velocity is zero, the damping term is nullified, as the derivative of the error is also zero. Thus, the correlation is limited to the SE kernel of the stiffness term, as illustrated in Fig. 3c. When the input point velocity is not zero, damping term correlations arise. Figs. 3a, 3b, and 3d, 3e show both negative and positive values of $\dot{x}$, which determine the slope of the correlation around $x-x^{\prime}=0$, as the derivative of the error is proportional to $\dot{x}$.

\section{Conditional distributions of latent functionals}

Due to multivariate normality, the conditional distributions are also Gaussian distributions and computed in closed form. In this work and in HRI in general, the human motor intention, i.e., the desired trajectory $x_{d, i}(\boldsymbol{\theta})$ represents especially relevant information. From (12) and (19), the a priori joint distribution $\mathrm{P}\left(\boldsymbol{u}_{\text {int }, i}(\bar{\Xi}), x_{d, i}\left(\boldsymbol{\theta}_{*}\right) \mid \bar{\Xi}, \boldsymbol{\theta}_{*}\right)$ of observations of the interaction force $\boldsymbol{u}_{\text {int }, i}(\bar{\Xi})=\left\{u_{\text {int }, i, j}(\overline{\boldsymbol{\xi}})\right\}_{j=1}^{h}$ at inputs $\bar{\Xi}=\left\{\overline{\boldsymbol{\xi}}_{j}\right\}_{j=1}^{h}$ and the desired trajectory $x_{d, i}\left(\boldsymbol{\theta}_{*}\right)$ at test input $\boldsymbol{\theta}_{*}$ yields

$$
\begin{aligned}
& \mathrm{P}\left(\boldsymbol{u}_{\text {int }, i}(\bar{\Xi}), x_{d, i}\left(\boldsymbol{\theta}_{*}\right) \mid \bar{\Xi}, \boldsymbol{\theta}_{*}\right)= \\
& \mathcal{N}\left(\left[\begin{array}{c}
\boldsymbol{u}_{\text {int }, i}(\bar{\Xi}) \\
x_{d, i}\left(\boldsymbol{\theta}_{*}\right)
\end{array}\right] \mid\left[\begin{array}{c}
\mathrm{E}\left[\boldsymbol{u}_{\text {int }, i}(\bar{\Xi})\right] \\
\mu_{x_{d}, i}
\end{array}\right],\left[\begin{array}{ll}
K_{\text {int }} & C_{\text {int }} \\
C_{\text {int }}^{\top} & k_{x_{d}}^{* *}
\end{array}\right]\right),
\end{aligned}
$$

where $K_{\text {int }}=k_{\text {int }}\left(\bar{\Xi}, \bar{\Xi}^{\prime}\right), C_{\text {int }}=\operatorname{Cov}\left[\boldsymbol{u}_{\text {int }, i}(\bar{\Xi}), x_{d, i}\left(\boldsymbol{\theta}_{*}\right)\right]$, and $k_{x_{d}}^{* *}=k_{x_{d}}\left(\boldsymbol{\theta}_{*}, \boldsymbol{\theta}_{*}\right)$. The covariance function is defined as

$$
\begin{aligned}
C_{\text {int }}= & \mu_{m, i} \frac{\partial^{2} k_{x_{d}}\left(\Theta, \boldsymbol{\theta}_{*}\right)}{\partial t^{2}}+\mu_{d, i} \frac{\partial k_{x_{d}}\left(\Theta, \boldsymbol{\theta}_{*}\right)}{\partial t} \\
& +\mu_{k, i} k_{x_{d}}\left(\Theta, \boldsymbol{\theta}_{*}\right)
\end{aligned}
$$

with the task-specific input parameter inputs $\Theta=\left\{\boldsymbol{\theta}_{j}\right\}_{j=1}^{h}$. Application of Bayes' rule yields the conditional posterior

$$
\begin{gathered}
\mathrm{P}\left(x_{d, i}\left(\boldsymbol{\theta}_{*}\right) \mid \boldsymbol{u}_{\mathrm{int}, i}(\bar{\Xi}), \bar{\Xi}, \boldsymbol{\theta}_{*}\right)= \\
\mathcal{N}\left(x_{d, i}\left(\boldsymbol{\theta}_{*}\right) \mid \mu_{x_{d}, i}+C_{\mathrm{int}}^{\boldsymbol{\top}} K_{\mathrm{int}}^{-1}\left(\boldsymbol{u}_{\mathrm{int}, i}(\bar{\Xi})-\mathrm{E}\left[\boldsymbol{u}_{\mathrm{int}, i}(\bar{\Xi})\right]\right),\right. \\
\left.k_{x_{d}}^{* *}-C_{\mathrm{int}}^{\boldsymbol{\top}} K_{\mathrm{int}}^{-1} C_{\mathrm{int}}\right)
\end{gathered}
$$

The computation of conditional posteriors of alternative latent functionals is performed analogously. In the non-physical HRI variation, $\mathrm{E}\left[\boldsymbol{u}_{\mathrm{int}, i}(\bar{\Xi})\right]=\boldsymbol{u}_{\mathrm{int}, i}(\bar{\Xi})=\mathbf{0}$ and $K_{\mathrm{int}}$ is replaced by $\bar{K}_{\text {int }}=\bar{k}_{\text {int }}\left(\bar{\Xi}, \bar{\Xi}^{\prime}\right)$ with kernel $\bar{k}_{\text {int }}\left(\overline{\boldsymbol{\xi}}, \overline{\boldsymbol{\xi}}^{\prime}\right)$ of (22).

\section{Pseudocode application instructions}

The following pseudocode shows the general algorithm structure of the impedance-based GP model for physical HRI.

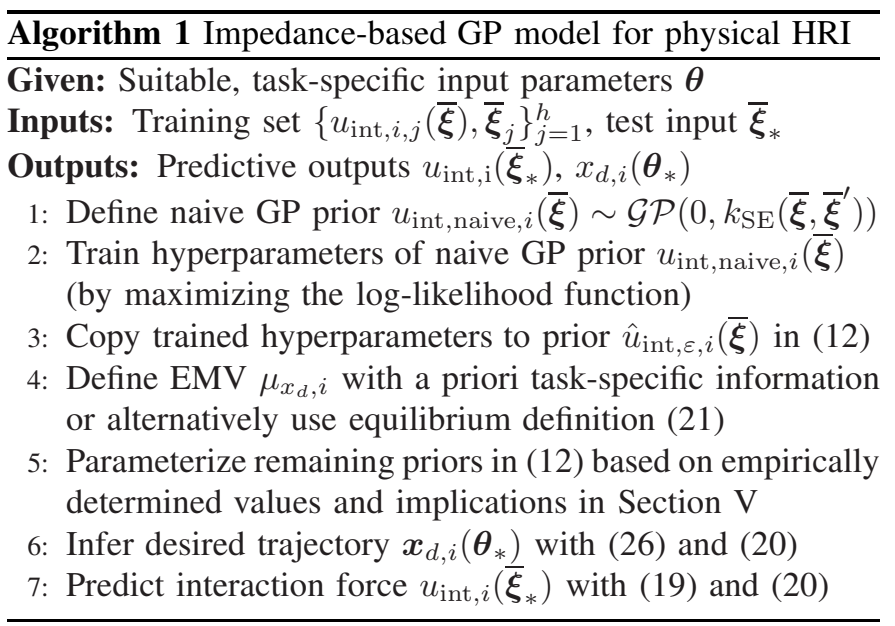

In the non-physical HRI variation, the inputs consist of the training set $\left\{0, \overline{\boldsymbol{\xi}}_{j}\right\}_{j=1}^{h}$ and the test input $\boldsymbol{\theta}_{*}$ and the outputs consist of the predictive output $x_{d, i}\left(\boldsymbol{\theta}_{*}\right)$. Steps $1,2,3$, and 7 are omitted and in step 6 , covariance function $k_{\text {int }}\left(\overline{\boldsymbol{\xi}}, \overline{\boldsymbol{\xi}}^{\prime}\right)$ of (20) is replaced by covariance function $\bar{k}_{\text {int }}\left(\overline{\boldsymbol{\xi}}, \overline{\boldsymbol{\xi}}^{\prime}\right)$ of (22). 


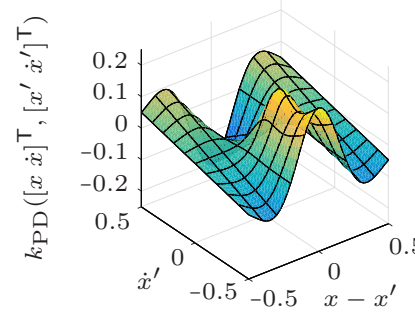

(a) $\dot{x}=-0.2$

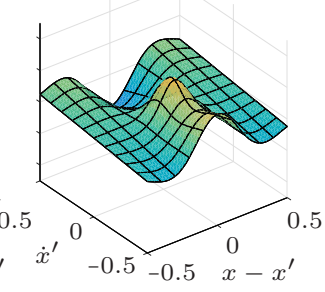

(b) $\dot{x}=-0.1$

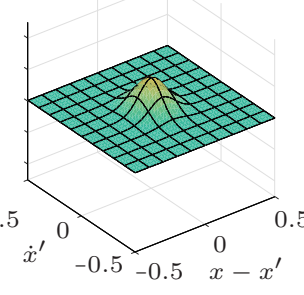

(c) $\dot{x}=0$

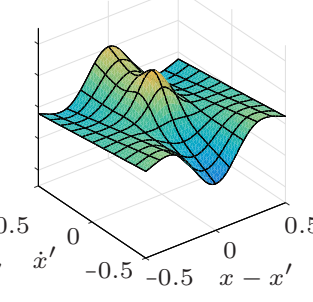

(d) $\dot{x}=0.1$

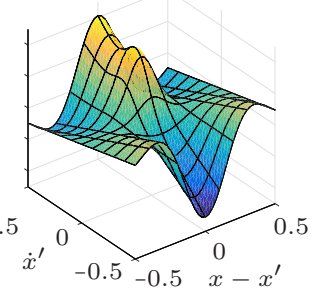

(e) $\dot{x}=0.2$

Fig. 3. Covariance functions of a PD kernel with $\theta=x$. The hyperparameters are $\{1,(0.2)\}_{x_{d}},\{0.1,(0.10 .1)\}_{d}$, and $\{0.1,(0.10 .1)\}_{k}$. The EMVs are $\mu_{d}=1.5 \mathrm{Ns} / \mathrm{m}, \mu_{k}=1 \mathrm{Nm}$, and $\mu_{x_{d}}=0 \mathrm{~m}$.

\section{PRIOR PARAMETERIZATION}

The prior parameters of the priors in (12) are composed of the EMVs $\mu_{*, i}$ and the hyperparameters of the SE kernels in (23), i.e., the signal variances $\sigma_{*, i}^{2}$ and the length scales $\Lambda_{*, i}$. Only the variable $m_{i}$ poses an exception. Due to the lack of a covariance function, it is defined only by the EMV $\mu_{m, i}$ and the signal variance $\sigma_{m, i}^{2}$. The parameters of prior $\hat{u}_{\mathrm{int}, \varepsilon, i}(\overline{\boldsymbol{\xi}})$ do not require manual definition, as its EMV is zero and its hyperparameters are trained by maximizing the log-likelihood function of a naive GP prior. In order to facilitate proper parameterization of the remaining priors in (12), an overview of estimated human arm impedance characteristics is presented and subsequently, resulting implications are discussed.

\section{A. Human arm impedance characteristics}

While there is a large body of work on estimation of human arm impedance, the results are often presented qualitatively or graphically using inertia, damping, and stiffness ellipses [9]. Of the few studies that present quantitative results, many estimate in joint space to address joint-specific impedance control by the CNS [28]. In this work, we instead focus on the Cartesian space representation due to practical applications in robotics [7], [8]. Table I shows an overview of estimated impedance characteristics in postural and non-postural tasks. In the former, a desired posture is maintained and in the latter, a desired movement is performed. The stiffness values lie in the approximate range of $10-1000 \mathrm{~N} / \mathrm{m}$ and are lower during non-postural tasks. The damping and inertia values lie in the approximate ranges of $5-100 \mathrm{Ns} / \mathrm{m}$ and $0.05-4 \mathrm{~kg}$. The estimation of damping is considered highly variable [48]. Some inertia values in Table I, which are much lower than the arm mass of a typical adult (approximately $2-4 \mathrm{~kg}$ [49]), suggest that inertia is particularly prone to estimation errors. This is plausible, as arm acceleration is often subject to high levels of noise. The positive correlation between joint damping and joint stiffness is visible in the values in Table I, which supports the statement that its effects can be partially incorporated by suitable definition of the EMVs $\mu_{d, i}$ and $\mu_{k, i}$. Furthermore, the low SDs of non-postural values in Table I (apart from the values of [35], which, due to the welding accuracy requirements, is similar to a postural task) support the consideration of low variances for the approximation in (13).

\section{B. Implications for prior parameterization}

The EMVs $\mu_{m, i}, \mu_{d, i}$, and $\mu_{k, i}$ should be defined based on the values in Table I. While the inertia EMV $\mu_{m, i}$ only depends on the limb kinematics, for the EMVs $\mu_{m, i}$ and $\mu_{d, i}$, the task description should be considered. Attention should be paid to correct definition of the principal movement axis.

Due to the strong dependence on task type and dimensions, only qualitative recommendations for the length scales $\Lambda_{*, i}$ and the signal variances $\sigma_{*, i}^{2}$ are possible. In general, the larger a length scale, the smaller the variation of the mean in the GP posterior. The expectation of a certain degree of smoothness of the desired trajectory $x_{d, i}(\boldsymbol{\theta})$ implies comparatively large length scales $\Lambda_{x_{d}, i}$. As the feedback component defined by damping $d_{i}(\boldsymbol{\xi})$ and stiffness $k_{i}(\boldsymbol{\xi})$ generates restoring torques

TABLE I

ESTIMATED IMPEDANCE CHARACTERISTICS. POSTURAL: $\boldsymbol{x}_{1}, \boldsymbol{x}_{2}$, AND $\boldsymbol{x}_{3}$ ARE SAGITTAL, TRANSVERSAL, AND VERTICAL AXES, RESPECTIVELY. NON-POSTURAL: $\boldsymbol{x}_{1}, \boldsymbol{x}_{2}$, AND $\boldsymbol{x}_{3}$ ARE PRINCIPAL MOVEMENT, LATERAL, AND VERTICAL AXES, RESPECTIVELY. PRESENTED IN MEAN (SD).

\begin{tabular}{|c|c|c|c|c|c|c|c|c|c|c|c|c|c|c|c|c|c|c|}
\hline \multirow[b]{2}{*}{ Study description } & \multicolumn{6}{|c|}{ Inertia [kg] } & \multicolumn{6}{|c|}{ Damping [Ns/m] } & \multicolumn{6}{|c|}{ Stiffness $[\mathrm{N} / \mathrm{m}]$} \\
\hline & \multicolumn{2}{|c|}{$x_{1}$} & \multicolumn{2}{|c|}{$x_{2}$} & \multicolumn{2}{|c|}{$x_{3}$} & \multicolumn{2}{|c|}{$x_{1}$} & \multicolumn{2}{|c|}{$x_{2}$} & \multicolumn{2}{|c|}{$x_{3}$} & \multicolumn{2}{|c|}{$x_{1}$} & \multicolumn{2}{|c|}{$x_{2}$} & \multicolumn{2}{|c|}{$x_{3}$} \\
\hline [43] postural & 1.54 & $(0.07)$ & 1.00 & $(0.08)$ & - & - & 10.7 & $(0.9)$ & 13.3 & $(1.2)$ & - & - & 117 & (14) & 236 & (27) & - & - \\
\hline [44] postural & 0.28 & $(0.15)$ & 0.39 & $(0.21)$ & 0.25 & $(0.13)$ & 35.3 & $(23.8)$ & 84.1 & $(52.2)$ & 40.1 & $(21.4)$ & 337 & (190) & 748 & $(281)$ & 303 & (209) \\
\hline [45] postural & 0.99 & $(0.07)$ & 1.42 & $(0.20)$ & - & - & 6.8 & $(0.8)$ & 14.3 & (1.4) & - & - & 27 & (4) & 91 & (6) & - & - \\
\hline [46] postural & 2.02 & $(0.39)$ & 1.60 & $(0.33)$ & - & - & 6.6 & (1.6) & 25.3 & (7.3) & - & - & 89 & (24) & 264 & (92) & - & - \\
\hline [47] postural & 0.75 & $(0.15)$ & 0.40 & $(0.10)$ & - & - & 9.9 & $(2.6)$ & 53.5 & (8.5) & - & - & 40 & (16) & 346 & (68) & - & - \\
\hline [35] welding - novice & 0.70 & $(0.30)$ & 2.40 & $(0.60)$ & 0.90 & $(0.40)$ & 15.0 & $(8.0)$ & 32.0 & $(13.0)$ & 12.0 & $(8.0)$ & 341 & (67) & 411 & (188) & 280 & (108) \\
\hline [35] welding - expert & 0.60 & $(0.20)$ & 2.80 & $(0.80)$ & 0.70 & $(0.20)$ & 19.0 & $(5.0)$ & 44.0 & (14.0) & 17.0 & $(5.0)$ & 423 & $(232)$ & 539 & (200) & 315 & $(150)$ \\
\hline [33] catching - static & 1.36 & $(0.09)$ & - & - & - & - & 20.6 & (2.1) & - & - & - & - & 67 & (34) & - & - & - & - \\
\hline [33] catching - ready & 1.44 & $(0.18)$ & $\ldots$ & - & - & - & 23.0 & (2.9) & - & - & - & - & 116 & (60) & - & - & - & - \\
\hline [36] teleoperation & 0.85 & & 4.03 & - & 0.68 & - & 12.9 & - & 9.2 & - & 17.6 & - & 122 & - & 108 & - & 81 & - \\
\hline [37] stylus grasping & 0.32 & $(0.15)$ & 0.48 & $(0.15)$ & 0.24 & $(0.13)$ & 5.9 & $(2.2)$ & 5.9 & (1.4) & 20.1 & $(5.1)$ & 105 & (28) & 133 & (29) & 366 & (106) \\
\hline [34] tennis - static & 1.79 & $(0.10)$ & - & - & - & - & 19.8 & (1.7) & - & - & - & - & 116 & (44) & - & - & - & - \\
\hline [34] tennis - ready & 1.84 & $(0.11)$ & - & - & - & - & 21.6 & (2.1) & - & - & - & - & 158 & (49) & - & - & - & - \\
\hline
\end{tabular}


that compensate deviations from the desired trajectory $x_{d, i}(\boldsymbol{\theta})$, the respective length scales $\Lambda_{d, i}$ and $\Lambda_{k, i}$ should be smaller than $\Lambda_{x_{d}, i}$. Otherwise, the model would misinterpret observed deviations as part of the desired trajectory $x_{d, i}(\boldsymbol{\theta})$. The trained hyperparameters of prior $\hat{u}_{\mathrm{int}, \varepsilon, i}(\overline{\boldsymbol{\xi}})$, which are obtained by maximizing the log-likelihood function of a naive GP prior, can serve as reference points: length scales $\Lambda_{x_{d}, i}$ should be larger and length scales $\Lambda_{d, i}$ and $\Lambda_{k, i}$ should be smaller.

For the definition of the signal variance $\sigma_{x_{d}, i}^{2}$, it should be considered that (18) does not only contain the desired trajectory $x_{d, i}(\boldsymbol{\theta})$, but also the corresponding time derivatives. As the variance increases with differentiation, a large signal variance $\sigma_{x_{d}, i}^{2}$ would result in considerable variance for the overall model. Consequently, the impedance contributions would "disappear" in the variance. In order to avoid this, the signal variance $\sigma_{x_{d}, i}^{2}$ should be defined sufficiently small. Due to similar reasons and the loss of accuracy caused by the second-order statistical characterization in (13), the signal variances $\sigma_{d, i}^{2}$ and $\sigma_{k, i}^{2}$ should also be sufficiently small.

Application of GP priors with SE covariance functions allows for derivation of theory concerning upper bounds of prediction errors. In [50], a probability bound for an upper bound of the prediction error is derived using the reproducing kernel Hilbert space norm. In [51], an upper bound for the mean square prediction error of misspecified GP models is derived by solving a pseudo-concave optimization problem. As in depth discussion of such theories lies outside the scope of this work, we instead provide quantitative examples for the qualitative parameter recommendations. Their impact on the results can be inferred from the validation with simulated data.

\section{SIMULATION}

In this section, the model is validated using a simulated model of the human arm. The simulated desired trajectory enables validation of the intention estimation capabilities and direct control over the simulated impedance characteristics allows for assessment of the effects of prior parameterization. In order to analyze how differences between the simulated impedance characteristics and the prior parameters affect the prediction performance, the damping and stiffness EMVs are both varied within plausible intervals. In order to consider the highly task-specific nature of human motor behavior in realistic HRI and demonstrate the versatility and adaptability of the model, it is validated both for a time-dependent $(\boldsymbol{\theta}=t)$ and a configuration-dependent $(\boldsymbol{\theta}=\boldsymbol{x})$ desired trajectory. All priors in (12) are defined by SE kernels. The prediction performance is assessed with the standardized mean squared error (SMSE) and the mean standardized log loss (MSLL). In the SMSE, the squared residuals of predicted and observed interaction forces $u_{\mathrm{int}, i}^{*}\left(\overline{\boldsymbol{\xi}}_{*, j}\right)$ and $u_{\mathrm{int}, i}\left(\overline{\boldsymbol{\xi}}_{*, j}\right)$ are averaged over the test inputs $\bar{\Xi}_{*}=\left\{\overline{\boldsymbol{\xi}}_{*, j}\right\}_{j=1}^{h}$ and normalized with the variance of the respective test outputs $\boldsymbol{u}_{\mathrm{int}, i}\left(\bar{\Xi}_{*}\right)$ :

$$
\mathrm{SMSE}=\frac{1}{n} \sum_{i=1}^{n}\left[\frac{1}{h} \sum_{j=1}^{h} \frac{\left(u_{\mathrm{int}, i}^{*}\left(\overline{\boldsymbol{\xi}}_{*, j}\right)-u_{\mathrm{int}, i}\left(\overline{\boldsymbol{\xi}}_{*, j}\right)\right)^{2}}{\operatorname{Var}\left[\boldsymbol{u}_{\mathrm{int}, i}\left(\bar{\Xi}_{*}\right)\right]}\right] .
$$

The MSLL is obtained from the negative log probability, standardized by subtracting the log probability of a Gaussian

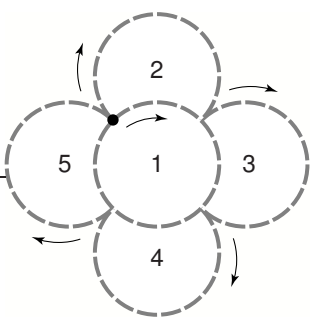

Fig. 4. Reference trajectory. Starting at the black dot, the arrows and numbers indicate movement direction and execution order, respectively.

with mean and variance of the training data $\boldsymbol{u}_{\text {int }, i}(\bar{\Xi})$ :

$$
\begin{array}{r}
\text { MSLL }=\frac{1}{n} \sum_{i=1}^{n}\left[\frac { 1 } { h } \sum _ { j = 1 } ^ { h } \left[-\log \mathrm{P}\left(u_{\mathrm{int}, i}^{*}\left(\overline{\boldsymbol{\xi}}_{*, j}\right) \mid \boldsymbol{u}_{\mathrm{int}, i}(\bar{\Xi})\right)\right.\right. \\
\left.\left.-\log \mathcal{N}\left(u_{\mathrm{int}, i}^{*}\left(\overline{\boldsymbol{\xi}}_{*, j}\right) \mid \mathrm{E}\left[\boldsymbol{u}_{\mathrm{int}, i}(\bar{\Xi})\right], \operatorname{Var}\left[\boldsymbol{u}_{\mathrm{int}, i}(\bar{\Xi})\right]\right)\right]\right]
\end{array}
$$

Analogous to the calculation of the SMSE, the MSLL is also determined by averaging over the test inputs $\bar{\Xi}_{*}[15]$.

\section{A. Human arm model \& simulation design}

A two-link rigid-body trajectory is simulated with the arm model of [52] using Matlab (Mathworks Inc.). The model is selected, because it is structurally more complex than our GP-based model and derives a two-dimensional trajectory from simulated muscle activities that provide physiologically plausible impedance characteristics. The model simulates the muscle tension $\boldsymbol{m}_{\mathrm{imp}}$ due to mechanical impedance as

$$
\begin{gathered}
\boldsymbol{m}_{\mathrm{imp}}=D_{\lambda} \dot{\boldsymbol{e}}_{\lambda}+K_{\lambda} \boldsymbol{e}_{\lambda}, \\
D_{\lambda}=1 / 12 K_{\lambda}, \quad K_{\lambda}=\left(1+0.035 \boldsymbol{a}_{\mathrm{CNS}}\right) K_{0},
\end{gathered}
$$

where $D_{\lambda}, K_{\lambda}$, and $e_{\lambda}$ are damping, stiffness, and error at muscular level, respectively, $\boldsymbol{a}_{\mathrm{CNS}}$ is the neural control signal, and $K_{0}$ is an intrinsic stiffness parameter. Two different stiffness profiles with $K_{0}=3360$ for low stiffness and $K_{0}=16800$ for high stiffness are each simulated at $500 \mathrm{~Hz}$. The simulated damping and stiffness are presented in Table II. The reference trajectory is displayed in Fig. 4. It consists of a circle with radius $0.04 \mathrm{~m}$ and four attached arcs that each subtend 270 degrees. The same reference trajectory is repeated three times without pause at a rate of $2.618 \mathrm{rad} / \mathrm{s}$ to form the complete data set. As the reference trajectory in Fig. 4 does not possess a distinct principal movement axis, the coordinates $x_{1}$ and $x_{2}$ refer to the sagittal and coronal planes, respectively. For the same reason, from this point on and without loss of generality, the impedance matrices $M(\boldsymbol{x}), D(\boldsymbol{\xi})$, and $K(\boldsymbol{\xi})$ are defined to be isotropic. For clarity, the index $i$ of priors

\begin{tabular}{|c|c|c|c|c|}
\hline \multirow{2}{*}{ Simulation } & \multicolumn{2}{|c|}{ Damping [Ns/m] } & \multicolumn{2}{|c|}{ Stiffness $[\mathbf{N} / \mathrm{m}]$} \\
\hline & $x_{1}$ & $x_{2}$ & $x_{1}$ & $x_{2}$ \\
\hline Low & 9.89 (1.47) & $12.40 \quad(0.73)$ & $118.7 \quad(17.6)$ & $148.4 \quad(20.7)$ \\
\hline High stiffness & $48.07 \quad(6.20)$ & $60.29 \quad(7.55)$ & 576.8 (74.4) & 723.5 (90.6) \\
\hline
\end{tabular}
$m_{i}, d_{i}(\boldsymbol{\xi})$, and $k_{i}(\boldsymbol{\xi})$ in (12) and the associated EMVs $\mu_{*, i}$, length scales $\Lambda_{*, i}$, and signal variances $\sigma_{*, i}^{2}$ is omitted.

TABLE ॥

SimUlated dAMPING AND STIFFNESS. PRESENTEd IN MEAN (SD). 


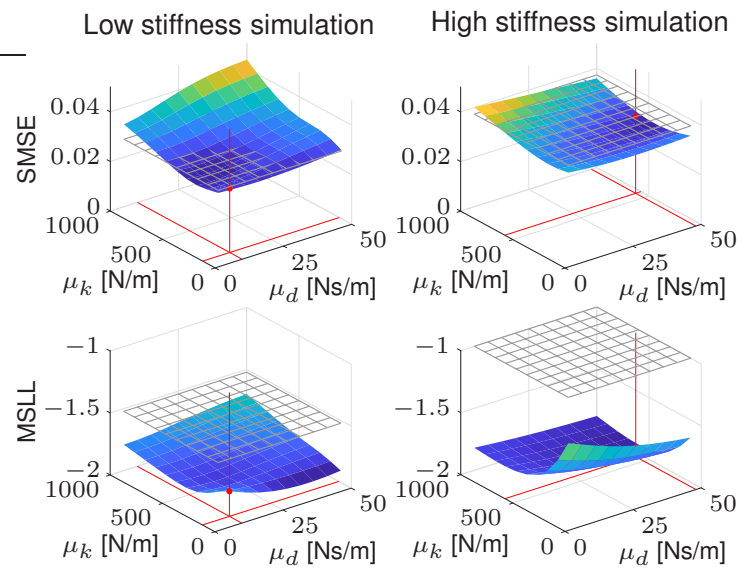

Fig. 5. Prediction performance with $\boldsymbol{\theta}=\boldsymbol{x}$. The red lines indicate the mean values of the simulated $x_{1}$ impedance values and the grey grid represents the performance of the naive GP prior.

\section{B. Configuration-dependent desired trajectory}

We consider (19) with a configuration-dependent desired trajectory $(\boldsymbol{\theta}=\boldsymbol{x})$ and a priori equilibrium definition (21). For simplicity, priors $m, d(\boldsymbol{\xi})$, and $k(\boldsymbol{\xi})$ are defined constant and deterministic, i.e., $\sigma_{m}^{2}=\sigma_{d}^{2}=\sigma_{k}^{2}=0$. The prediction performance of the model is evaluated with respect to a naive GP prior $u_{\text {int }, i}(\overline{\boldsymbol{\xi}}) \sim \mathcal{G} \mathcal{P}\left(0, k_{\mathrm{SE}}\left(\overline{\boldsymbol{\xi}}, \overline{\boldsymbol{\xi}}^{\prime}\right)\right)$. The training sets are obtained by downsampling the simulated trajectories to $0.2 \mathrm{~Hz}$ and the validation sets are obtained by downsampling the same trajectories to $10 \mathrm{~Hz}$. The hyperparameters of the naive model are trained by maximizing the log-likelihood and the resulting length scales and signal variances are also used to parameterize prior $\hat{u}_{\mathrm{int}, \varepsilon, i}(\overline{\boldsymbol{\xi}})$. The inertia EMV $\mu_{m}=3 \mathrm{~kg}$ and the damping and stiffness EMVs $\mu_{d} \in[5,50] \mathrm{Ns} / \mathrm{m}$ and $\mu_{k} \in[100,1000] \mathrm{N} / \mathrm{m}$. The hyperparameters of the desired trajectory are $\left\{10^{-6},(0.20 .2)\right\}_{x_{d}}$ and $\sigma_{\mathrm{N}}^{2}=10^{-4}$. In the second half of the analysis, the intention estimation capabilities of the model are evaluated via the inferred desired trajectory. The evaluation is conducted for the simulated trajectory of the high stiffness simulation, downsampled to $25 \mathrm{~Hz}$, and the results are presented for two sets of low $\left(\mu_{k}=100, \mu_{d}=5\right)$ and high $\left(\mu_{k}=800, \mu_{d}=40\right)$ stiffness and damping EMVs.

1) Behavior prediction performance: Fig. 5 presents the prediction performance results with respect to the naive model in terms of SMSE and MSLL. The SMSE for the low stiffness simulation shows that the impedance model outperforms the naive model over the low stiffness and low damping ranges, where the EMVs are close to the simulated values in Table II. The prediction performance is significantly decreased in the high stiffness and high damping ranges. In terms of the MSLL, the prediction performance increases throughout the whole stiffness and damping ranges and it reaches its maximum in the low stiffness and low damping ranges. The SMSE for the high stiffness simulation displays overall improved performance, especially for stiffness EMVs in the mid-range and a high damping EMV that is close to the simulated values in Table II. A similar dependency is found in the MSLL results. For low stiffness values, the impedance model still outperforms the naive model, but performance is reduced due to high variance, which is considered in the calculation of the MSLL.
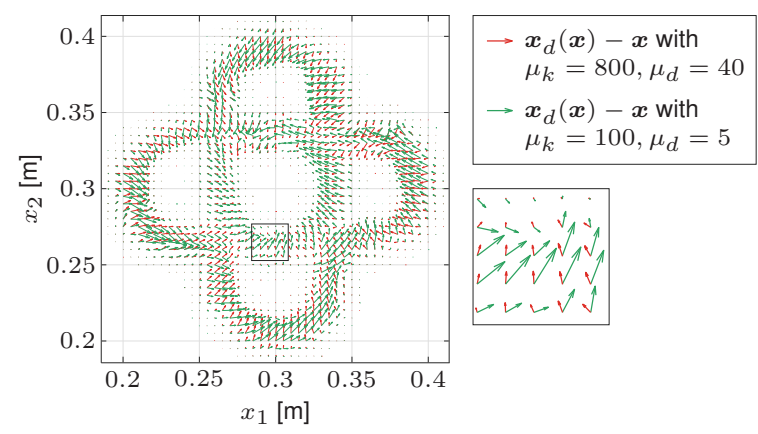

Fig. 6. Inference of the desired trajectory $\boldsymbol{x}_{\boldsymbol{d}}(\boldsymbol{x})$ with $\boldsymbol{\theta}=\boldsymbol{x}$ for the high stiffness simulation. The results are presented in terms of $\boldsymbol{x}_{d}(\boldsymbol{x})-\boldsymbol{x}$.

2) Intention estimation capabilities: Fig. 6 demonstrates the inference of the latent desired trajectory, as defined in (26). The arrows represent length and orientation of the differences between the input states $\boldsymbol{x}$ and the inferred states of the desired trajectory $\boldsymbol{x}_{d}(\boldsymbol{x})$. The model with high EMVs expects lower deviations due to higher tracking accuracy. Thus, the inferred state is persistently located in the proximity of the associated input state. In contrast, the model with low EMVs expects higher deviations due to lower tracking accuracy.

\section{Time-dependent desired trajectory}

Here, we consider (19) with a time-dependent desired trajectory $(\boldsymbol{\theta}=t)$ and a priori equilibrium definition (21). Parameterization and design are identical to Section VI-B, apart from the following differences: the error terms $\ddot{e}_{i}(t)$, $\dot{e}_{i}(t)$, and $e_{i}(t)$ and the estimated interaction force $\hat{u}_{\mathrm{int}, \varepsilon, i}(t)$ are defined as functions of time, such that, according to (10), the interaction force $u_{\text {int }, i}(t) \sim \mathcal{G P}\left(0, k_{\text {int }}\left(t, t^{\prime}\right)\right)$. Therefore, the naive GP prior is defined as $u_{\text {int }, i}(t) \sim \mathcal{G P}\left(0, k_{\mathrm{SE}}\left(t, t^{\prime}\right)\right)$. The training sets are obtained by downsampling the simulated trajectories to $3 \mathrm{~Hz}$ and the hyperparameters of the desired trajectory are $\left\{10^{-6},(0.3)\right\}_{x_{d}}$ and $\sigma_{\mathrm{N}}^{2}=10^{-4}$.

1) Behavior prediction performance: Fig. 7 presents the prediction performance results. The SMSE for the low stiffness simulation shows that the impedance model outperforms the naive model over the whole stiffness and damping ranges, especially for low stiffness and damping EMVs, which are close to the simulated values in Table II. In terms of the MSLL, the prediction performance increases throughout the whole stiffness and damping ranges and it reaches its maximum in the mid-stiffness and low damping ranges. The SMSE for the high stiffness simulation displays overall improved performance, especially for the high damping and high stiffness ranges, when the EMVs are close to the simulated values in Table II. A similar dependency is found in the MSLL results.

2) Intention estimation capabilities: Fig. 8 illustrates the inference of the latent desired trajectory, as defined in (26). It contains the inferred desired trajectories, the corresponding SDs, and the reference trajectory. The model with high EMVs expects lower deviations due to higher tracking accuracy. Consequently, the inferred desired trajectory possesses low variance. As the EMVs are close to the simulated values of the high stiffness simulation, the model represents the simulated dynamics with high accuracy. The model with 

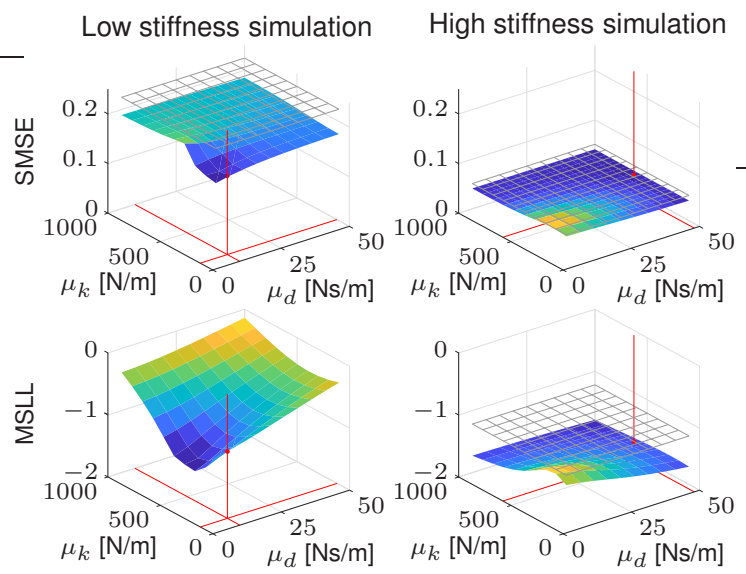

Fig. 7. Prediction performance with $\boldsymbol{\theta}=\boldsymbol{t}$. The red lines indicate the mean values of the simulated $x_{1}$ impedance values and the grey grid represents the performance of the naive GP prior. The naive GP prior MSLL in the low stiffness simulation is 14.14 and thus omitted for clarity.

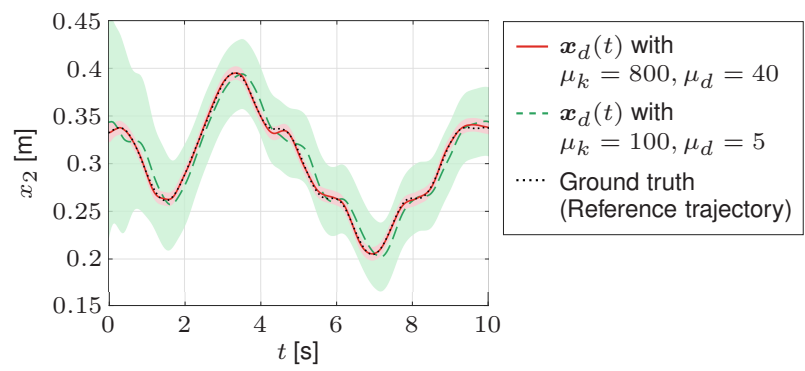

Fig. 8. Inference of the desired trajectory $x_{d}(t)$ with $\theta=t$ for the high stiffness simulation. The shaded areas are the SDs of the desired trajectory $x_{d}(t)$ and are amplified for clarity ( \pm 5 SD for the model with low EMV values, \pm 10 SD for the model with high EMV values).

low EMVs expects higher deviations due to lower tracking accuracy. As the EMVs are not close to the simulated values, the simulated dynamics are represented with less accuracy. The inferred desired trajectory possesses higher variance and deviates significantly more from the reference trajectory.

The configuration- and time-dependent analyses in this section validate the intention estimation capabilities and the superior prediction performance of the model. In addition to being a positive result, the latter represents a secondary means of validation for the inferred desired trajectories. Additionally, the impact of proper prior parameterization is demonstrated.

\section{EXPERIMENT}

In order to assess the prediction performance with real data and envisage applications in HRI, an experiment with human participants is performed. The experiment design is based on the simulation design. However, the impedance priors are not defined constant and deterministic and the EMVs are not varied within plausible intervals. Instead, the number of training points is decreased to determine the effects of training data sparsity. In order to assess the generalization capabilities of the model, two different scenarios are evaluated, in which the training and validation sets are located in identical or different regions of the workspace. In order to demonstrate the versatility and adaptability of the model, it is evaluated for a configuration- and velocity-dependent desired trajectory. The prediction performance is quantified by the SMSE.

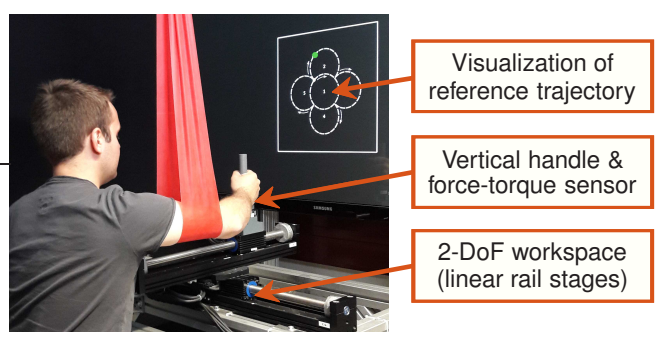

Fig. 9. Participant interacting with the apparatus during the experiment.

\section{A. Apparatus \& data processing}

The apparatus in Fig. 9 consists of two linear, orthogonally aligned single rail stages (Copley Controls Thrusttube Module) that span a 2-DoF workspace of $\pm 0.15 \mathrm{~m}$. Each module provides position data with a precision of $1 \mu \mathrm{m}$. On top of the upper module, a vertical handle with a 6-DoF force-torque sensor (JR3-67M25) is mounted. Visual feedback is supplied with the Psychophysics Toolbox [53] and shown on a computer screen at participant eye level. Haptic interaction by means of force input is enabled by an admittance control scheme

$$
M_{a} \ddot{\boldsymbol{x}}+D_{a} \dot{\boldsymbol{x}}=\boldsymbol{u}_{\mathrm{int}},
$$

where $M_{a}=\operatorname{diag}\{5,5\} \mathrm{kg}$ and $D_{a}=\operatorname{diag}\{15,15\} \mathrm{Ns} / \mathrm{m}$ are the admittance inertia and damping. Precise position rendering is provided by high gain PD control, which is implemented in Matlab/Simulink and executed on a RT-preempt Linux system with a real-time kernel. The sample rate is $4 \mathrm{kHz}$ and the Thrusttube Module inputs are downsampled to $2 \mathrm{kHz}$ due to hardware limitations. All signals are filtered using a 5 -th order Savitzky-Golay filter with a cut-off frequency of $20 \mathrm{~Hz}$.

\section{B. Participants \& experiment design}

A total of 20 participants (16 male, 4 female) volunteered to take part in this experiment. All participants had normal or corrected-to-normal vision and were right handed. The average age was 26.95 (4.41) years. Informed written consent was obtained from all participants before experiment participation. The research ethics were obtained from the ethics committee at the Technical University of Munich (project no. 205/14).

The participants were seated in front of the apparatus and instructed to grasp the handle with their right hand. Their arm was constrained to horizontal movements by a sling attached to the ceiling. The reference trajectory and the current position of the cart were visualized on the computer screen (Fig. 9). The participants were instructed to first move to the starting point and then follow the trajectory at their own pace (Fig. 4). Each participant completed 15 repetitions without pause.

\section{Parameterization}

Here, we consider (19) with a configuration and velocitydependent desired trajectory $\left(\boldsymbol{\theta}=\left[\boldsymbol{x}^{\top}, \dot{\boldsymbol{x}}^{\top}\right]^{\top}\right)$ and a priori equilibrium definition (21). For computational feasibility, we set all jerk-related terms in (18) to zero. The training sets are obtained from data of repetitions $6-10$. It is assumed that after five repetitions the participants have had enough time to familiarize with the apparatus. The validation sets are 

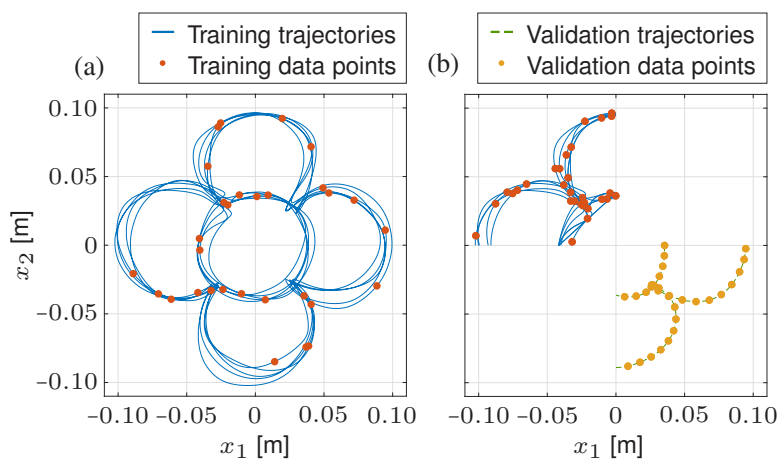

Fig. 10. Participant data. (a) Identical regions with exemplary 30 point training set. (b) Different regions with exemplary 30 point training and validation sets (number of validation points reduced for clarity).

obtained from data of repetitions $11-15$. In order to ensure comprehensive coverage of the workspace, each repetition is validated separately. The number of training points is increased from 2 to 50 and there are 200 validation points. Before the evaluation, the training and validation points are uniformly distributed among the sets. In each evaluation, a training point is randomly selected and added to the existing training set. The evaluations are repeated 100 times for each of the five validation repetitions $(11-15)$ and the results are averaged. Figs. 10a and 10b show the identical and different workspace regions scenarios, respectively. In the former, all sets contain data from all workspace quadrants. In the latter, training sets only contain data from Quadrant 2 and validation sets only contain data from Quadrant 4. The hyperparameters of prior $\hat{u}_{\text {int }, \varepsilon, i}(\overline{\boldsymbol{\xi}})$ are defined by maximum log-likelihood training of a naive GP prior. The EMVs are $\mu_{m}=2 \mathrm{~kg}$, $\mu_{d}=20 \mathrm{Ns} / \mathrm{m}$, and $\mu_{k}=100 \mathrm{~N} / \mathrm{m}$. They are defined based on the principal movement axis values in Table I: $\mu_{d}$ is the approximate mean of the damping values and $\mu_{k}$ is based on the lower stiffness values, as they represent the most plausible values for the observed dynamics. The hyperparameters are

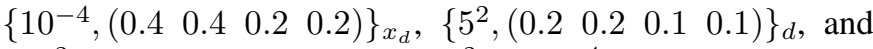

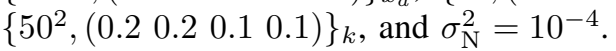

\section{Evaluation}

Fig. 11 shows the averaged SMSE results over the number of training points $N$. The mean SMSEs rapidly decrease with an increase of $N$. Although the initial value for different workspace regions is significantly increased, the values for large $N$ are almost identical. The mean SMSE converges to zero for identical workspace regions and to a marginally larger value for different workspace regions. This decrease in prediction performance is plausible, as the validation points are located in previously unobserved regions of the input space.

The results show that decreased prediction performance due to training data sparsity only arises for very small numbers of training points $N$. Although this decrease increases with the distance between training and validation points, given a reasonable number of training points, the model nonetheless provides reliable predictions in previously unobserved regions of the input space. This reliable prediction performance of the model for real data also serves as an indirect means of validation for its intention estimation capabilities.

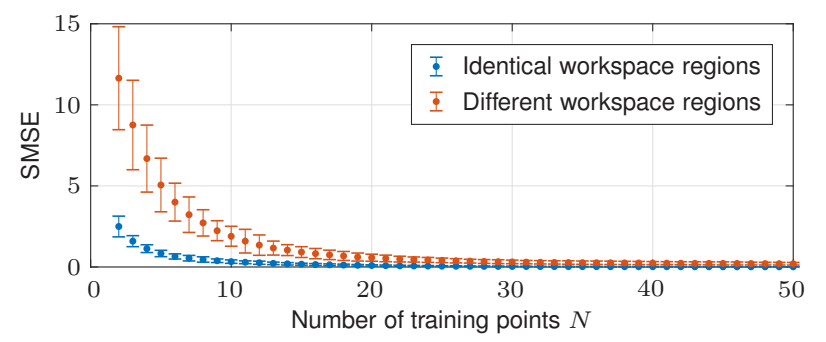

Fig. 11. Averaged SMSE results. The error bars each indicate $\pm \mathbf{5}$ SD.

\section{DISCUSSION}

In this work, we derive an impedance-based GP model. The derivation of this Bayesian model only requires that the desired behavior can be described by a twice differentiable, well-defined function $\boldsymbol{q}_{d}(\boldsymbol{\theta})$. The parameters $\boldsymbol{\theta}$ are defined by task-specific input parameters such as $\theta=t$ for a transient desired trajectory $\boldsymbol{q}_{d}(t)$. We demonstrate desirable intention estimation capabilities and superior prediction performance for different parameterizations of the desired trajectory $\boldsymbol{q}_{d}(\boldsymbol{\theta})$. In the experiment, $\boldsymbol{\theta}=\left[\boldsymbol{x}^{\top}, \dot{\boldsymbol{x}}^{\top}\right]^{\top}$. We exclude repetitions $1-5$ from the data, assuming that after five repetitions participants have had enough time to familiarize with the apparatus and possess a desired trajectory $\boldsymbol{x}_{d}(\boldsymbol{x}, \dot{\boldsymbol{x}})$. It should be noted that this trajectory does not have to be and likely is not completely identical to the visual reference trajectory. Nonetheless, our assumption is supported by desirable prediction performance, indicated by low averaged SMSE results, both for previously observed and unobserved regions of the input space.

The results obtained with simulated and real physical HRI data also support the general applicability of the approximation of diagonality for impedance matrices $M(\boldsymbol{x}), D(\boldsymbol{\xi})$, and $K(\boldsymbol{\xi})$. Nonetheless, in future work, we aim to extend the method to physiologically accurate multi-dimensional treatment of (8), which would require definition of the non-diagonal elements by additional GPs. Each of these GPs would require proper prior parameterization, including the corresponding EMVs, for which empirically determined data would first need to be obtained. The additional product terms would need to be incorporated in (14) and (15). More importantly, symmetry properties of the matrices would require definition of an additional covariance function that correlates the respective GP with the interaction forces along the $\mathrm{x}$ - and $\mathrm{y}$-axes. Physiological plausibility of the model is also slightly reduced by omitting the positive correlation of damping $d_{i}(\boldsymbol{\xi})$ and stiffness $k_{i}(\boldsymbol{\xi})$ in the prior definitions (12). It can however be partially included in the model through suitable definition of the EMVs $\mu_{d, i}$ and $\mu_{k, i}$. In the experiment, we define low values for $\mu_{d, i}$ and $\mu_{k, i}$, compared to the significantly higher values of postural tasks. If the observed behavior is in the proximity of the expected behavior, correlational characteristics of the conditional posteriors of $d_{i}(\boldsymbol{\xi})$ and $k_{i}(\boldsymbol{\xi})$ are to be expected. If the observed behavior deviates greatly from the expected behavior, the conditional posteriors may lose correlation, which may result in reduced accuracy of the inferred desired trajectory $\boldsymbol{x}_{d}\left(\boldsymbol{\theta}_{*}\right)$ and the predicted interaction forces $\boldsymbol{u}_{\text {int }}\left(\overline{\boldsymbol{\xi}}_{*}\right)$. Such behavioral and potentially correlational deviations are indicated by decreased model confidence levels. 


\section{CONCLUSION}

We present a novel method for modeling human motor behavior in physical and non-physical HRI that considers complex tasks with trajectory tracking requirements, in which the human arm dynamics can be modeled by a mechanical impedance that tracks a latent desired trajectory. The results of simulated and real physical HRI data show strong support of our method, demonstrating superior prediction performance as well as desirable intention estimation and generalization capabilities. In future work, we will explore online application as well as combination with established impedance estimation methods for continuous parameter adaptation.

\section{REFERENCES}

[1] L. Marchal-Crespo and D. J. Reinkensmeyer, "Review of control strategies for robotic movement training after neurologic injury," J. Neuroeng. Rehabil., vol. 6, no. 1, p. 20, 2009.

[2] K. Anam and A. Al-Jumaily, "Active exoskeleton control systems: State of the art," Procedia Eng., 2012.

[3] S. Schaal, "Is imitation learning the route to humanoid robots?" Trends Cognit. Sci., vol. 3, no. 6, pp. 233-242, 1999.

[4] F. Ficuciello et al., "Variable impedance control of redundant manipulators for intuitive human-robot physical interaction," IEEE Trans. Robot., vol. 31, pp. 850-863, 2015.

[5] D. W. Franklin and D. M. Wolpert, "Computational mechanisms of sensorimotor control," Neuron, vol. 72, no. 3, pp. 425-442, 2011.

[6] C. M. Harris and D. M. Wolpert, "Signal-dependent noise determines motor planning," Nature, vol. 394, no. 6695, p. 780, 1998.

[7] E. Gribovskaya et al., "Motion learning and adaptive impedance for robot control during physical interaction with humans," in Proc. IEEE Int. Conf. Robot. Autom., 2011, pp. 4326-4332.

[8] V. Duchaine and C. Gosselin, "Safe, stable and intuitive control for physical human-robot interaction," in Proc. IEEE Int. Conf. Robot. Autom., 2009, pp. 3383-3388.

[9] T. Flash and N. Hogan, "The coordination of arm movements: an experimentally confirmed mathematical model," J. Neurosci., vol. 5, no. 7, pp. 1688-1703, 1985.

[10] Y. Maeda et al., "Human-robot cooperative manipulation with motion estimation," in Proc. IEEE/RSJ Int. Conf. Intell. Robots Syst., vol. 4, 2001, pp. 2240-2245.

[11] B. Corteville et al., "Human-inspired robot assistant for fast point-topoint movements," in Proc. IEEE ICRA, 2007, pp. 3639-3644.

[12] S. Miossec and A. Kheddar, "Human motion in cooperative tasks: moving object case study," in Proc. IEEE Int. Conf. Robot. Biomim., 2009, pp. 1509-1514.

[13] A. Jha and S. S. Chiddarwar, "Robot programming by demonstration using teleoperation through imitation," Industrial Robot, vol. 44, no. 2, pp. 142-154, 2017.

[14] A. Billard et al., "Robot programming by demonstration," in Springer handbook of robotics. Springer, 2008, pp. 1371-1394.

[15] C. E. Rasmussen, "Gaussian processes in machine learning," in Advanced lectures on machine learning. Springer, 2004, pp. 63-71.

[16] J. Kocijan et al., "Dynamic systems identification with gaussian processes," Math. Comput. Model. Dyn. Syst., vol. 11, pp. 411-424, 2005.

[17] E. Solak et al., "Derivative observations in gaussian process models of dynamic systems," in Adv. Neural Inf. Proc. Syst., 2003, pp. 1057-1064.

[18] J. Wang et al., "Gaussian process dynamical models for human motion," IEEE Trans. Pattern Anal. Mach. Intell., vol. 30, pp. 283-298, 2008.

[19] Z. Wang et al., "Probabilistic modeling of human movements for intention inference," in Proc. Robot. Sci. Syst., 2012.

[20] M. Álvarez et al., "Linear latent force models using gaussian processes," IEEE Trans. Pattern Anal. Mach. Intell., vol. 35, pp. 2693-2705, 2013.

[21] L. Castellanos et al., "A multivariate gaussian process factor model for hand shape during reach-to-grasp movements," Statistica Sinica, vol. 25, no. 1 , p. $5,2015$.

[22] J. R. Medina et al., "Risk-sensitive optimal feedback control for haptic assistance," in Proc. IEEE Int. Conf. Robot Autom, 2012, pp. 1025-1031.

[23] A. Van Der Vaart and H. Van Zanten, "Information rates of nonparametric gaussian process methods," J. Mach. Learn. Res., vol. 12, pp. 2095-2119, 2011.

[24] M. Kawato, "Internal models for motor control and trajectory planning," Curr. Opin. Neurobiol., vol. 9, no. 6, pp. 718-727, 1999.
[25] H. Gomi and M. Kawato, "Human arm stiffness and equilibrium-point trajectory during multi-joint movement," Biol. Cybern., vol. 76, no. 3, pp. $163-171,1997$.

[26] E. Burdet et al., "The central nervous system stabilizes unstable dynamics by learning optimal impedance," Nature, vol. 414, no. 6862, pp. 446-449, 2001

[27] J. M. Dolan et al., "Dynamic and loaded impedance components in the maintenance of human arm posture," IEEE Trans. Syst., Man, Cybern., vol. 23, pp. 698-709, 1993.

[28] H. Gomi and R. Osu, "Task-dependent viscoelasticity of human multijoint arm and its spatial characteristics for interaction with environments," J. Neurosci., vol. 18, no. 21, pp. 8965-8978, 1998.

[29] J. R. Medina et al., "Impedance-based gaussian processes for predicting human behavior during physical interaction," in Proc. IEEE Int. Conf. Robot. Autom., 2016, pp. 3055-3061.

[30] A. C. Lo et al., "Robot-assisted therapy for long-term upper-limb impairment after stroke," N. Engl. J. Med., vol. 362, no. 19, pp. 1772 1783, 2010.

[31] L. Peternel et al., "Unifying speed-accuracy trade-off and cost-benefit trade-off in human reaching movements," Front. Hum. Neurosci., vol. 11, p. 615, 2017.

[32] K. P. Tee et al., "A model of force and impedance in human arm movements," Biol. Cybern., vol. 90, no. 5, pp. 368-375, 2004.

[33] Y. Tanaka et al., "Task readiness impedance in human arm movements for virtual ball-catching task," in Proc. IEEE Annu. Conf. Ind. Electron. Soc., vol. 1, 2003, pp. 478-483.

[34] T. Tsuji et al., "Analysis of mechanical impedance in human arm movements using a virtual tennis system," Biol. Cybern., vol. 91, no. 5, pp. 295-305, 2004.

[35] M. S. Erden and A. Billard, "Hand impedance measurements during interactive manual welding with a robot," IEEE Trans. Robot., vol. 31, pp. 168-179, 2015.

[36] J. E. Speich et al., "Modeling the human hand as it interacts with a telemanipulation system," Mechatronics, vol. 15, pp. 1127-1142, 2005

[37] M. J. Fu and M. C. Cavusoglu, "Human-arm-and-hand-dynamic model with variability analyses for a stylus-based haptic interface," IEEE Trans. Syst., Man, Cybern., vol. 42, pp. 1633-1644, 2012.

[38] M. Álvarez and N. Lawrence, "Sparse convolved gaussian processes for multi-output regression," in Adv. Neural Inf. Proc. Syst, 2009, pp. 57-64.

[39] E. Snelson et al., "Warped gaussian processes," in Adv. Neural Inf. Proc. Syst., 2004, pp. 337-344.

[40] G. W. Bohrnstedt and A. S. Goldberger, "On the exact covariance of products of random variables," J. Am. Stat. Assoc., vol. 64, no. 328, pp. 1439-1442, 1969.

[41] J. Shawe-Taylor and N. Cristianini, Kernel methods for pattern analysis. Cambridge university press, 2004.

[42] D. Duvenaud et al., "Structure discovery in nonparametric regression through compositional kernel search," in Proc. Int. Conf. Mach. Learn., vol. 28, no. 3. PMLR, 2013, pp. 1166-1174.

[43] T. Tsuji et al., "Human hand impedance characteristics during maintained posture," Biol. Cybern., vol. 72, no. 6, pp. 475-485, 1995.

[44] P. K. Artemiadis et al., "Human arm impedance: characterization and modeling in 3d space," in Proc. IEEE/RSJ Int. Conf. Intell. Robots Syst., 2010, pp. 3103-3108

[45] M. Dyck and M. Tavakoli, "Measuring the dynamic impedance of the human arm without a force sensor," in Proc. IEEE Int. Conf. Rehabil. Robot., 2013, pp. 1-8.

[46] P. H. Chang et al., "Stochastic estimation of human arm impedance using robots with nonlinear frictions: an experimental validation," IEEE/ASME Trans. Mechatronics, vol. 18, pp. 775-786, 2013.

[47] S. Wang et al., "Human hand impedance characteristics during reaching movements," in Proc. IEEE Int. Conf. Biomed. Eng. Inform., vol. 3, 2011, pp. 1282-1286.

[48] E. J. Perreault et al., "Multijoint dynamics and postural stability of the human arm," Exp. Brain Res., vol. 157, no. 4, pp. 507-517, 2004.

[49] C. E. Clauser et al., "Weight, volume, and center of mass of segments of the human body," Aerospace Research Laboratory, Tech. Rep., 1969.

[50] N. Srinivas et al., "Information-theoretic regret bounds for gaussian process optimization in the bandit setting," IEEE Trans. Inf. Theory, vol. 58, pp. 3250-3265, 2012.

[51] T. Beckers et al., "Mean square prediction error of misspecified gaussian process models," in Proc. IEEE Conf. Decis. Control, 2018.

[52] D. W. Franklin et al., "Cns learns stable, accurate, and efficient movements using a simple algorithm," J. Neurosci., vol. 28, no. 44, pp. 11 165-11 173, 2008.

[53] D. H. Brainard, "The psychophysics toolbox," Spatial Vision, vol. 10, pp. 433-436, 1997. 